\title{
On Vector Bimeasures (*).
}

\author{
KARI YLINEN ('Turku, Finland)
}

Summary. - See Introduction.

\section{1. - Introduction.}

The notion of a bimeasure in a bilinear funetional sense has been studied by M. Morse and W. Transue in a series of papers (cf. e.g. [12-16]) and E. Thomas [20]. The bimeasures considered by these authors are (separately or, equivalently, jointly) continuous sealar valued bilinear forms defined on the Cartesian product of the spaces $\nVdash\left(S_{1}\right)$ and $\mathscr{K}\left(S_{2}\right)$ of continuous functions with compact support on the locally compact Hausdorff spaces $S_{1}$ and $S_{2}\left(K\left(S_{j}\right)\right.$ being equipped with the usual locally convex inductive limit topology). The approach in the present article, where (vector) bimeasures are defined as (vector valued) separately $\sigma$-additive functions on the Cartesian product of two $\sigma$-algebras, is motivated by a desire to find an analogue of the Riesz (-Markov-Kakutani) representation theorem and its vector generalization (cf. [1], [6]) which says that the weakly compact operators from $C_{0}(\mathbb{S})$ to $X$ (where $X$ is a Banach space, $S$ is a locally compact Hausdorff space, and $O_{0}(S)$ is the space of continuous scalar functions on $S$ vanishing at infinity, equipped with the supremum norm) are via integration in a bijective correspondence with the regular $X$-valued Borel vector measures on $S$. In the scalar case a Riesz type representation theorem in some form seems to be part of the folklore of the subject (see e.g. E. Thomas's review of [5] in Math. Reviews, 46, no. 9285), though we haven't seen any proof in the generality involving bounded bilinear forms on $C_{0}\left(S_{1}\right) \times C_{0}\left(S_{2}\right)$ for arbitrary locally compact Hausdorff spaces $S_{1}$ and $S_{2}$. In this connection it may be observed that the early history of the representation of bilinear forms (see [7]) is closely related (even temporally) to F. Riesz's pioneering work on the representation of linear forms.

Our main representation theorem is Theorem 6.9. That result resembles the vector generalization of the Riesz representation theorem. The counterpart of a weakly compact operator is here a bounded bilinear operator $B: C_{0}\left(S_{1}\right) \times C_{0}\left(S_{2}\right) \rightarrow X$ whose canonical extension (i.e. the unique separately weak*-to-weak* continuous

(*) Entrata in Redazione il 30 marzo 1977. 
extension $B_{e}: C_{0}\left(S_{1}\right)^{\prime \prime} \times C_{0}\left(S_{2}\right)^{\prime \prime} \rightarrow X^{\prime \prime}$ of $B$; such an extension is shown to always exist) maps $O_{0}\left(S_{1}\right)^{\prime \prime} \times C_{0}\left(S_{2}\right)^{\prime \prime}$ into $X$. These bilinear operators are shown to be via integration (this term is made precise in Section 5 ) in a bijective correspondence with the mappings $\beta: \mathfrak{B}_{1} \times \mathfrak{B}_{2} \rightarrow X\left(\mathfrak{B}_{j}\right.$ is the Borel $\sigma$-algebra of $\left.\mathcal{S}_{j}\right)$ for which $\beta(E, \cdot): \mathfrak{B}_{2} \rightarrow X$ and $\beta(\cdot, F): \mathfrak{B}_{1} \rightarrow X$ are regular vector measures for all $E \in \mathfrak{B}_{1}$, $F \in \mathfrak{B}_{2}$. Such a mapping $\beta$ is called a (separately regular) vector bimeasure.

In general, a vector bimeasure in our terminology is a separately $\sigma$-additive mapping $\beta: \Sigma_{1} \times \Sigma_{2} \rightarrow X$ where $\Sigma_{1}$ and $\Sigma_{2}$ are $\sigma$-algebras. In Section 5 we develop a theory of integration of a pair of functions with respect to a vector bimeasure in much greater generality than would be necessary for the representation theorem (where the consideration of continuous functions vanishing at infinity suffices). The semivariation of a vector bimeasure is discussed in Section 4. In Section 3 we deal with some aspects of the theory of vector measures needed in the sequel.

Once we have the correspondence between the separately regular vector bimeasures $\beta: \mathscr{B}_{1} \times \mathfrak{B}_{2} \rightarrow X$ and the class of bilinear operators $B: C_{0}\left(S_{1}\right) \times C_{0}\left(S_{2}\right) \rightarrow X$ specified above, another approach to the integration of pairs of functions, more closely reminiscent of the method of Morse and Transue, suggests itself. In Section 7 it is shown, roughly, that a technique based on the use of the vector measures corresponding to the weakly compact operators

$$
B(\cdot, k): C_{0}\left(S_{1}\right) \rightarrow X \quad \text { and } \quad B(h, \cdot): C_{0}\left(S_{2}\right) \rightarrow X
$$

for $k \in O_{0}\left(S_{2}\right), h \in C_{0}\left(S_{1}\right)$, in place of the vector measures $\beta(\cdot, F)$ and $\beta(E, \cdot), F \in \mathscr{B}_{2}$, $E \in \mathfrak{B}_{1}$, leads to a theory equivalent to the one developed in Section 5 if and only if $X$ does not contain any isomorphic copy of $e_{0}$.

\section{2. - Preliminaries and notation.}

The notation introduced here will remain fixed throughout the paper. The scalar field can be either $\boldsymbol{R}$ or $\boldsymbol{C}$; we use (consistently) the common notation $\boldsymbol{K}$ for both. We let $X$ always be a Banach space over $\boldsymbol{K}$. The normed dual of $X$ is denoted by $X^{\prime}$, and we write $x^{\prime}(x)=\left\langle x^{\prime}, x\right\rangle=\left\langle x, x^{\prime}\right\rangle$ for $x \in X, x^{\prime} \in X^{\prime}$. By definition, $\sigma\left(X, X^{\prime}\right)$ is the weak topology on $X$ and $\sigma\left(X^{\prime}, X\right)$ is the weak topology on $X^{\prime}$. The norm in $X$ and in $X^{\prime}$ is denoted by $|\cdot|$, and we write e.g. $X_{1}^{\prime}=\left\{x^{\prime} \in X^{\prime}:\left|x^{\prime}\right| \leqslant 1\right\}$. The notation $|\cdot|$ is also used for the norm of a bounded linear or bilinear operator; thus e.g. $|B|=\sup \left\{|B(y, z)|: y \in Y_{1}, z \in Z_{1}\right\}$, if $Y$ and $Z$ are also normed spaces and $B: Y \times Z \rightarrow X$ is a bounded bilinear operator. The adjoint of a bounded linear operator $T$ is denoted by $T^{\prime}$.

In most questions pertaining to integration theory we follow [6]. For $j=1,2$, $S_{j}$ (resp. $S$ ) is a non-empty set, $\Sigma_{j}$ (resp. $\Sigma$ ) is a $\sigma$-algebra of subsets of $S_{j}$ (resp. $\$$ ). A $\sigma$-additive (or, equivalently, weakly $\sigma$-additive $[6, \mathrm{p} .318]$ ) set function $\mu: \Sigma \rightarrow X$ is called a vector measure. The semivariation (in the sense of $[6, p .320]$ ) of a vector 
measure $\mu: \Sigma \rightarrow X$ is denoted by $\|\mu\| ;\|\mu\|$ is a bounded nonnegative-valued $\sigma$-subadditive function on $\Sigma$. If here $X=\boldsymbol{K}, \mu$ is called a measure, and $\|\mu\|$ agrees with the total variation of $\mu$. We usually denote the total variation of a measure $\mu: \Sigma \rightarrow K$ by $v(\mu)$ and its value for a set $E \in \Sigma$ by $v(\mu, E)$. We let $c a(S, \Sigma)$ denote the Banach space [6, p. 161] of the measures $\mu: \Sigma \rightarrow K$ equipped with the norm $|\mu|=v(\mu, S)$ The $\mu$-measurability of a function $f: \Sigma \rightarrow K$ for $\mu \in e a(S, \Sigma)$ is defined in [6, p. 106] and its $\Sigma$-measurability in $[6, p .240]$. Recall that $f$ is $\mu$-measurable if and only if it is $\Sigma^{*}$-measurable, where $\Sigma^{*}$ is the Lebesgue extension of $\Sigma$ relative to $\mu[6, p .148]$. If $E \subset S, \chi_{E}: S \rightarrow\{0,1\}$ denotes the characteristic function of $E$.

\section{3. - Measurability and integrability with respect to vector measures.}

In this section we present some material on vector measures needed in the study of vector bimeasures. Throughout, $\mu: \Sigma \rightarrow X$ is a vector measure. For each $x^{\prime} \in X^{\prime}$, the measure $E \mapsto\left\langle x^{\prime}, \mu(E)\right\rangle, E \in \Sigma$, is denoted by $x^{\prime} \mu$. An examination of the steps leading to Corollary 2.4 in $[1$, p. 294] yields the version of that result appearing in part $(b)$ of the following lemma. Part $(a)$ is well known and easy to prove.

LEMMA 3.1. - (a) $\|\mu\|(E)=\sup _{x^{\prime} \in X_{1}^{\prime}} v\left(x^{\prime} \mu, E\right)$ for all $E \in \Sigma$. (b) There exists a positive measure $\lambda \in e a(S, \Sigma)$ such that

(1) $\lambda(E) \leqslant\|\mu\|(E)$ for all $E \in \Sigma$, and

(2) $\lim _{\lambda(E) \rightarrow 0}\|\mu\|(E)=0$.

Let $D \subset X_{1}^{\prime}$ be such that for a fixed constant $C>0$ we have $\sup \left\{\left|\left\langle x^{\prime}, x\right\rangle\right|: x^{\prime} \in D\right\} \geqslant$ $\geqslant C|x|$ for all $x \in X$. Then a measure satisfying (1) and (2) oan be chosen to be the sum of an absolutely convergent series $\sum_{n=1}^{\infty} a_{n} v\left(x_{n}^{\prime} \mu\right)$ where $a_{13}$ is a positive number and
$x_{n}^{\prime} \in D$ for all $n \in N$.

The expressions $\mu$-null set, $\mu$-almost everywhere (abbreviated $\mu$-a.e.) and $\mu$-measurable funetion will have the same meanings as in $[6, p .322]$. The following result is analogous to Proposition 2.17 in [20, p. 95].

THEOREM 3.2. - Let $D \subset X_{1}^{\prime}$ be as in Lemma 3.1. Then a funetion $f: S \rightarrow \boldsymbol{K}$ is $\mu$-measurable if and only if $f$ is $x^{\prime} \mu$-measurable for every $x^{\prime} \in D$.

Proof. - Let $a_{n}>0, x_{n}^{\prime} \in D$ for $n \in N$ and the measure $\lambda=\sum_{n=1}^{\infty} a_{n} v\left(x_{n}^{\prime} \mu\right)$ be as in Lemma 3.1. Denote by $\Sigma^{*}$ (resp. $\Sigma_{x^{*}}^{*}$ ) the Lebesgue extension of $\Sigma$ relative to $\lambda$ (resp. $\left.x^{\prime} \mu\right)[6$, p. 143]. We show that

$$
\Sigma^{*}=\bigcap_{x^{\prime} \in D} \Sigma_{\infty^{\prime}}^{*}
$$


Choose $A \in \bigcap_{x^{\prime} \in D} \Sigma_{x^{\prime}}^{*} \subset \bigcap_{n=1}^{\infty} \Sigma_{x_{n}^{\prime}}^{*}$. For each $n \in N$ there are sets $E_{n} \in \Sigma, N_{n} \subset \mathbb{S}$ and $M_{n} \in \Sigma$ such that $A=E_{n} \cup N_{n}, N_{n}^{\prime} \subset M_{n}$ and $v\left(x_{n}^{\prime} \mu, M_{n}\right)=0\left[6\right.$, p. 142]. Write $E=\bigcup_{n=1}^{\infty} E_{n}$, $N=\bigcap_{n=1}^{\infty} N_{n}, M=\bigcap_{n=1}^{\infty} M_{n}$. Then $A=E \cup N, E \in \Sigma, N \subset M$, and $M \in \Sigma$. Since $v\left(x_{n}^{\prime} \mu, M\right) \leqslant$ $\leqslant v\left(x_{n}^{\prime} \mu, M_{n}\right)=0$ for all $n \in N, \lambda(M)=\sum_{n=1}^{\infty} a_{n} v\left(x_{n}^{\prime} \mu, M\right)=0$. Thus $A \in \Sigma^{*}$. Conversely, let $B \in \Sigma^{*}$, so that $B=F \cup P$, where $F \in \Sigma$ and $P \subset R$ for some $R \in \Sigma$ with $\lambda(R)=0$. Then $v\left(x^{\prime} \mu, R\right) \leqslant\|\mu\|(R)=0$ for all $x^{\prime} \in D$, and so $B \in \bigcap_{x^{\prime} \in D} \Sigma_{x^{\prime}}^{*}$. Since the $\mu$-measurability of $f$ is equivalent to its $\Sigma^{*}$-measurability, and the $x^{\prime} \mu$-measurability of $f$ is equivalent to its $\Sigma_{x^{\prime}}^{*}$-measurability, the assertion follows from (1).

A $\Sigma$-measurable function $f: S \rightarrow \boldsymbol{K}$ which assumes only a finite number of values is called a $\Sigma$-simple function. The definition of the integral $\int_{E F} f d \mu, E \in \Sigma$, of a $\Sigma$-simple function $f$ is obvious $[6$, p. 322]. The following definition is used e.g. in $[6$, p. 323].

Definition 3.3. - A function $f: B \rightarrow K$ is said to be $\mu$-integrable, if there is a sequence $\left(f_{n}\right)$ of $\Sigma$-simple functions converging to $f \mu$-a.e. and such that the sequence $\left(\int_{E} f_{n} d \mu\right)$ is (norm) convergent in $X$ for each $E \in \Sigma$. We then write

$$
\lim _{n \rightarrow \infty} \int_{E} f_{n} d \mu=\int_{E} f d \mu=\int_{E} f(s) \mu(d s), \quad E \in \Sigma .
$$

REMraRK 3.4. - Every $\mu$-integrable function $f: S \rightarrow \boldsymbol{K}$ is $\mu$-measurable [6, p. 150]. The integral is an unambiguously defined element of $X[6, p .323]$. We shall use without explicit mention the well-known fact that, in case $X=\boldsymbol{K}$, Definition 3.3 is equivalent to Definition 17 in [6, p. 112]. A proof could be given by using, for one direction, Corollary 3 in $[6$, p. 145], and Fgoroff's theorem and the Vitali-HahnSaks theorem for the other.

The following theorem is closely related to some results in [11], but we give a complete proof, because in our case $f$ need not be $\Sigma$-measurable.

Theoream 3.5. - Let $D \subset X_{1}^{\prime}$ and $C>0$ be as in Lemma 3.1. A function $f: S \rightarrow K$ is $\mu$-integrable if and only if the following two conditions hold:

(i) $f$ is $x^{\prime} \mu$-integrable for each $x^{\prime} \in D$;

(ii) $\lim _{n \rightarrow \infty} \sup _{x^{\prime} \in \mathcal{D}} \int_{E_{n}}|f(s)| v\left(x^{\prime} \mu, d s\right)=0$ whenever the sets $E_{n} \in \Sigma$ satisfy $E_{n+1} \subset E_{n}, n \in N$, and $\bigcap_{n=1}^{\infty} E_{n}=\emptyset$.

Proof. - Suppose first that $f$ is $\mu$-integrable. Clearly, (i) holds [6, p. 324]. Write $\nu(E)=\int_{E} f d \mu, E \in \Sigma$. Then $\nu: \Sigma \rightarrow X$ is a vector measure [6, p. 323]. If the sets $E_{n}$ 
are as in (ii), then

$$
\sup _{x^{\prime} \in D} \int_{E_{n}}|f(s)| v\left(x^{\prime} \mu, d s\right)=\sup _{x^{\prime} \in D} v\left(x^{\prime} v, E_{n}\right) \leqslant\|v\|\left(E_{n}\right) \rightarrow 0
$$

as $n \rightarrow \infty$ (see [6, p. 114] and use Lemma 3.1 or e.g. Theorem 1.3 in [11]). Conversely, assume (i) and (ii). Since $f$ is $\mu$-measurable by Remark 3.4 and Theorem 3.2, there is a $\Sigma$-measurable function $f_{0}: S \rightarrow K$ which agrees with $f \mu$-a.e. (see e.g. [19, p. 145]); in particular, (i) and (ii) hold for $f_{0}$. Denote $E_{n}=\left\{s \in S:\left|f_{0}(s)\right| \geqslant n\right\} \in \Sigma$, $n \in \boldsymbol{N}$. For each $n \in \boldsymbol{N}$ there is a $\Sigma$-simple function $f_{n}: S \rightarrow \boldsymbol{K}$ satisfying $f_{n}(s)=0$ for $s \in E_{n}$ and $\left|f_{n}(s)\right| \leqslant\left|f_{0}(s)\right|,\left|f_{n}(s)-f_{0}(s)\right|<1 / n$ for $s \in S \backslash E_{n}$. Then $\lim _{n \rightarrow \infty} f_{n}(s)=f_{0}(s)$, $s \in S$. If $x^{\prime} \in D$, we have for all $E \in \Sigma$,

$$
\left|\left\langle\int_{E E_{m}}\left(f_{n}-f_{m}\right) d \mu, x^{\prime}\right\rangle\right| \leqslant 2 \int_{E E_{m}}\left|f_{0}(s)\right| v\left(x^{\prime} \mu, d s\right)
$$

and so

$$
\left|\int_{E E_{m}}\left(f_{n}-f_{m}\right) d \mu\right| \leqslant \frac{2}{C} \sup _{x^{\prime} \in D} \int_{E E_{m}}\left|f_{0}(s)\right| v\left(x^{\prime} \mu, d s\right)
$$

If e.g. $n \geqslant m, E_{n} \subset E_{m}$, so that

$$
\left|f_{n}(s)-f_{m}(s)\right| \leqslant\left|f_{n}(s)-f_{0}(s)\right|+\left|f_{0}(s)-f_{m}(s)\right|<\frac{1}{n}+\frac{1}{m}, \quad s \in S \backslash E_{m} .
$$

Thus

$$
\begin{aligned}
\left|\int_{E} f_{n} d \mu-\int_{E} f_{m} d \mu\right| \leqslant & \left|\int_{E E_{m}}\left(f_{n}-f_{m}\right) d \mu\right|+ \\
& +\left|\int_{E \backslash E_{m}}\left(f_{n}-f_{m}\right) d \mu\right| \leqslant \frac{2}{C_{x^{\prime} \in D}} \sup _{E_{m}}\left|f_{0}(s)\right| v\left(x^{\prime} \mu, d s\right)+\|\mu\|(S)\left(\frac{1}{n}+\frac{1}{m}\right) .
\end{aligned}
$$
It follows that $\left(\int_{E} f_{n} d \mu\right)$ is a Cauchy sequence in $X$. By definition $f_{0}$, and hence $f$,
is $\mu$-integrable.

The following corollary is essentially (i.e. modulo Theorem 3.2) contained in Theorem 2.4 in [11].

COROLLARY 3.6. - A function $f: S \rightarrow K$ is $\mu$-integrable if and only if the following two conditions are satisfied:

(i) $f$ is $x^{\prime} \mu$-integrable for each $x^{\prime} \in X^{\prime}$; (ii) for each $E \in \Sigma$ there is $\nu(E) \in X$ (clearly unique) such that $\left\langle\nu(E), x^{\prime}\right\rangle=\int_{E} f d x^{\prime} \mu$
for all $x^{\prime} \in X^{\prime}$.

If this is the case, then $v(E)=\int_{E} f d \mu, E \in \Sigma$. 
Proof. - Assume (i) and (ii). Since $\nu: \Sigma \rightarrow X$ is weakly, hence strongly, $\sigma$-additive, condition (ii) of Theorem 3.5 holds when $D=X_{1}^{\prime}$ (see Theorem $20(a)$ in $[6, p .114]$ and use Lemma 3.1), so that $f$ is $\mu$-integrable. The converse part and the equation $\nu(E)=\int_{E} f d \mu$ follow from Theorem $8(f)$ in $[6$, p. 324].

Corollary 3.7. - If a function $f: S \rightarrow \boldsymbol{K}$ is integrable with respect to each of the vector measures $\mu: \Sigma \rightarrow X$ and $v: \Sigma \rightarrow X$, then $f$ is $(\mu+v)$-integrable, and $\int_{E} f d(\mu+v)=$ $=\int_{E} f d \mu+\int_{E} f d v$ for all $E \in \Sigma$.

Proof. - The above corollary reduces the proof to the standard scalar case.

\section{4. - The Banach space of vector bimeasures.}

Recall that $\Sigma_{j}$ is a $\sigma$-algebra of subsets of $S_{j} \neq \emptyset, j=1,2$.

Definition 4.1. - If $\beta: \Sigma_{1} \times \Sigma_{2} \rightarrow X$ is separately $\sigma$-additive, i.e. if $\beta(E, \cdot)$ and $\beta(\cdot, F)$ are vector measures for all $E \in \Sigma_{1}, E \in \Sigma_{2}$, then $\beta$ is called a vector $b i$ measure. In case $X=\boldsymbol{K}, \beta$ is simply called a bimeasure.

We shall define a norm in the space of vector bimeasures, and for that purpose we introduce the notion of semivariation. In Section 6 we need the concept also for separately (finitely) additive mappings, so the definition is formulated in that generality.

Defintition 4,2 , - A partition $\left(E_{k}\right)_{k=1}^{m}$ of a set $E \in \Sigma_{j}$ is called a $\Sigma_{j}$-partition, if $E_{k} \in \Sigma_{j}$ for all $k=1, \ldots, m$. Let $\beta: \Sigma_{1} \times \Sigma_{2} \rightarrow X$ be separately additive. For $E \in \Sigma_{1}$, $F \in \Sigma_{2}$, we let $\|\beta\|(E, F)$ denote the supremum of the numbers $\left|\sum_{k=1}^{m} \sum_{n=1}^{n} a_{k} b_{p} \beta\left(E_{k}, F_{n}\right)\right|$ where always $\left(E_{k}\right)_{k=1}^{m}$ is a $\Sigma_{1}$-partition of $E,\left(F_{p}\right)_{p=1}^{n}$ is a $\Sigma_{2}$-partition of $F$, and $a_{k}, b_{3} \in K,\left|a_{k}\right| \leqslant 1,\left|b_{p}\right| \leqslant 1$ for $k=1, \ldots, m, p=1, \ldots, n$. The extended real valued function $(E, F) \mapsto\|\beta\|(E, F)$ on $\Sigma_{1} \times \Sigma_{2}$ is called the semivariation of $\beta$.

Lemma 4.3. - If $\beta$ is as in the above definition, and $D \subset X_{1}^{\prime}$ is such that $|x|=$ $=\sup _{x^{\prime} \in \mathcal{D}}\left|\left\langle x, x^{\prime}\right\rangle\right|$ for all $x \in X$, then $\|\beta\|(E, F)=\sup _{x^{\prime} \in \mathcal{D}}\left\|x^{\prime} \circ \beta\right\|(E, F)$ for all $E \in \Sigma_{1}, F \in \Sigma_{2}$.

Proof. - If $\left(E_{k}\right)_{k=1}^{m},\left(F_{p}\right)_{y=1}^{n}$, and $a_{k}, b_{p}$ are as in Definition 4.2 , we have

$$
\sup _{x^{\prime} \in D}\left|\sum_{k=1}^{m} \sum_{p=1}^{n} a_{k} b_{p} x^{\prime} \circ \beta\left(E_{k}, F_{p}\right)\right|=\left|\sum_{k=1}^{m} \sum_{p=1}^{n} a_{k} b_{p} \beta\left(E_{k}, F_{p}\right)\right| .
$$

Thus the assertion follows from elementary properties of the supremum.

In the rest of this section we confine our attention to separately $\sigma$-additive functions, although the separately finitely additive case could be treated in an analogous way. 
THEoneMr 4.4. - Let $\beta: \Sigma_{1} \times \Sigma_{2} \rightarrow \boldsymbol{K}$ be a bimeasure, and denote $\beta_{1}(E)=\beta(E, \cdot)$ for each $E \in \Sigma_{1}$, and $\beta_{2}(F)=\beta(\cdot, F)$ for each $F \in \Sigma_{2}$. Then the mappings $\beta_{1}: \Sigma_{1} \rightarrow$ $\rightarrow e a\left(S_{2}, \Sigma_{2}\right)$ and $\beta_{2}: \Sigma_{2} \rightarrow e a\left(S_{1}, \Sigma_{1}\right)$ are vector measures, and $\|\beta\|\left(S_{1}, S_{2}\right)=\left\|\beta_{1}\right\|\left(S_{1}\right)=$ $=\left\|\beta_{2}\right\|\left(\mathcal{S}_{2}\right)$.

Proof. - To show that e.g. $\beta_{1}$ is a vector measure, let $\left(E_{k}\right)_{k=1}^{\infty}$ be a sequence of pairwise disjoint members of $\Sigma_{1}$. Since $\lim _{n \rightarrow \infty} \rho\left(\bigcup_{k=1}^{n} E_{k}, F\right)$ exists for all $F \in \Sigma_{2}$, the measures $\beta_{1}\left(\bigcup_{k=1}^{n} E_{k}\right), n \in N$, form a bounded sequence in $c a\left(S_{2}, \Sigma_{2}\right)$ (see Theorem 8 in $[6$, p. 309] and Lemma 5 in $[6$, p. 97]). From Theorem 5 in $[6$, p. 308] it follows that the sequence of the measures $\beta_{1}\left(\bigcup_{k=1}^{n} E_{k}\right)$ converges weakly in $c a\left(S_{2}, \Sigma_{2}\right)$ to $\beta_{1}\left(\bigcup_{k=1}^{\infty} E_{k}\right)$. Thus $\beta_{1}$ is weakly, hence strongly, $\sigma$-additive. Let now $\left(E_{k}\right)_{k=1}^{m}$ be a $\Sigma_{1}$-partition of $S_{1}$ and $\left(F_{p}\right)_{y=1}^{n}$ a $\Sigma_{a}$-partition of $S_{2}$, and suppose $a_{k}, b_{p} \in K,\left|a_{k}\right| \leqslant 1$, $\left|b_{p}\right| \leqslant 1, k=1, \ldots, m, p=1, \ldots, n$. Then

$$
\left|\sum_{p=1}^{n} b_{p}\left(\left(\sum_{k=1}^{m} a_{k} \beta_{1}\left(E_{k}\right)\right)\left(F_{p}\right)\right)\right|=\left|\sum_{k=1}^{m} \sum_{p=1}^{n} a_{k} b_{p} \beta\left(E_{k}, F_{p}\right)\right| .
$$

The supremum of all numbers obtainable in this way as the left hand side of this equation is easily seen to be $\left\|\beta_{1}\right\|\left(S_{1}\right)$, and so $\left\|\beta_{1}\right\|\left(S_{1}\right)=\|\beta\|\left(S_{1}, S_{2}\right)$. The equality $\|\beta\|\left(S_{1}, S_{2}\right)=\left\|\beta_{2}\right\|\left(S_{2}\right)$ is proved similarly.

THEOREMr 4.5. - (a) For any vector bimeasure $\beta: \Sigma_{1} \times \Sigma_{2} \rightarrow X$

$\sup \left\{|\beta(E, F)|: E \in \Sigma_{1}, F^{\prime} \in \Sigma_{2}\right\} \leqslant\|\beta\|\left(S_{1}, S_{2}\right) \leqslant 16 \sup \left\{\left|\beta\left(E, F^{\prime}\right)\right|: E \in \Sigma_{1}, F^{\prime} \in \Sigma_{2}\right\}<\infty$.

(b) The set of vector bimeasures $\beta: \Sigma_{1} \times \Sigma_{2} \rightarrow X$ is a Banach space with respect to the pointwise operations and the norm $\beta \mapsto\|\beta\|\left(S_{1}, S_{2}\right)$.

Proof. - (a) The first inequality follows at once from the definition. By the preceding theorem and Lemma 4 in $[6$, p. 320],

$$
\begin{aligned}
& \left\|x^{\prime} \circ \beta\right\|\left(\mathbb{S}_{1}, \mathbb{S}_{2}\right)=\left\|\left(x^{\prime} \circ \beta\right)_{1}\right\|\left(S_{1}\right) \leqslant 4 \sup _{E \in \Sigma_{1}} v\left(\left(x^{\prime} \circ \beta\right)_{1}(E), \mathbb{S}_{2}\right) \leqslant \\
& \quad \leqslant 4 \sup _{E \in \Sigma_{1}}\left(4 \sup _{F \in \Sigma_{2}} \mid\left\langle x^{\prime}, \beta(E, F)\right\rangle\right) \leqslant 16 \sup \left\{|\beta(E, F)|: E \in \Sigma_{1}, F \in \Sigma_{2}\right\}
\end{aligned}
$$

for all $x^{\prime} \in X_{1}^{\prime}$, and so the second inequality follows from Lemma 4.3. Finally $\left\|x^{\prime} \circ \beta\right\|\left(S_{1}, S_{2}\right)=\left\|\left(x^{\prime} \circ \beta\right)_{1}\right\|\left(S_{1}\right)$ is finite for all $x^{\prime} \in X^{\prime}$ by Theorem 4.4 and Lemma 4 in $\left[6\right.$, p. 320], i.e. $\sup \left\{\left|\left\langle x^{\prime}, \beta(E, F)\right\rangle\right|: E \in \Sigma_{1}, F \in \Sigma_{2}\right\}<\infty$. Alternatively, this follows directly from Theorem 8 in $[6$, p. 309]. By the uniform boundedness principle $\sup \left\{|\beta(E, F)|: E \in \Sigma_{1}, F \in \Sigma_{2}\right\}<\infty$. 
(b) It is elear that the vector bimeasures form a linear space. It is quickly verified that $\|\cdot\|\left(S_{1}, S_{2}\right)$ (which is finite by $\left.(a)\right)$ is a norm. To prove the completeness of the space, let $\left(\beta_{n}\right)$ be a Cauchy sequence of vector bimeasures. Then $\beta\left(E, F^{\prime}\right)=\lim _{n \rightarrow \infty} \beta_{n}(E, F)$ exists for all $E \in \Sigma_{1}, F \in \Sigma_{2}$, and the convergence is uniform on $\Sigma_{1} \times \Sigma_{2}$. A standard argument then shows that $\beta$ is separately $\sigma$-additive, and by $(a), \lim _{n \rightarrow \infty}\left\|\beta_{n}-\beta\right\|\left(S_{1}, S_{2}\right)=0$.

\section{5. - Integration with respect to a vector bimeasure.}

Throughout this section, $\beta: \Sigma_{1} \times \Sigma_{2} \rightarrow X$ is a vector bimeasure. Before considering the integration of a pair of functions with respect to $\beta$ we prove an auxiliary result.

LEMMA 5.1. - Let $f: \$_{1} \rightarrow \boldsymbol{K}$ be $\beta(\cdot, F)$-integrable for every $F \in \Sigma_{2}$. Then the set function $F \mapsto \int_{E} f d \beta(\cdot, F), F \in \Sigma_{2}$, is $\sigma$-additive for every $E \in \Sigma_{1}$.

Proof. - Let us first treat the special case where $X=K$. Consider the $\operatorname{ca}\left(S_{2}, \Sigma_{2}\right)$ valued vector measure $\beta_{1}$, i.e. $E \mapsto \beta(E, \cdot)$ (cf. Theorem 4.4). Let $\lambda \in c a\left(S_{1}, \Sigma_{1}\right)$ be a positive measure satisfying the conditions (1) and (2) of Lemma 3.1 relative to $\beta_{1}$. Each $F \in \Sigma_{2}$ determines a bounded linear functional $\chi_{F}^{\prime}$ on $c a\left(S_{2}, \Sigma_{2}\right)$ by $\left\langle\nu, \chi_{F}^{\prime}\right\rangle=$ $=v(F)$, and the set $D=\left\{\chi_{F}^{\prime}: F \in \Sigma_{2}\right\}$ has the property $\sup _{\varphi \in D}|\langle v, \varphi\rangle| \geqslant \frac{1}{4} v\left(v, S_{2}\right)$ for all $v \in c a\left(S_{2}, \Sigma_{2}\right)\left[6\right.$, p. 97]. Since $f$ is $\beta_{1}$-measurable by Theorem 3.2, i.e. $f$ is $\Sigma_{1}^{*}$-measurable where $\Sigma_{1}^{*}$ is the Lebesgue extension of $\Sigma_{1}$ relative to $\lambda$, there is as $\Sigma_{1}$-measurable function $f_{0}: S_{1} \rightarrow \boldsymbol{K}$ which agrees with $f \beta_{1}$-a.e. (and hence $\beta(\cdot, F)$-a.e. for each $\left.F^{f} \in \Sigma_{2}\right)[19, \mathrm{p} .145]$. There is a sequence $\left(f_{n}\right)$ of $\Sigma_{1}$-simple functions such that $\left.\left|f_{n}(s)\right| \leqslant \mid f_{0}(s)\right\}$ and $\lim _{n \rightarrow \infty} f_{n}(s)=f_{0}(s)$ for all $s \in \mathbb{S}_{1}$. As $f_{0}$ is $\beta(\cdot, F)$-integrable for each $F \in \Sigma_{2}$,

$$
\lim _{n \rightarrow \infty} \int_{E} f_{n} d \beta(\cdot, F)=\int_{E} f_{0} d \beta(\cdot, F)=\int_{E} f d \beta(\cdot, F) \quad \text { for all } F \in \Sigma_{2}
$$

by the Lebesgue dominated convergence theorem [6, p. 151]. Since the set function $F \mapsto \int_{\tilde{E}} f_{n} d \beta(\cdot, F), F \in \Sigma_{2}$, is $\sigma$-additive for each $n \in N$, Corollary 4 in $[6$, p. 160] shows that $F \mapsto \int_{E} f d \beta(\cdot, F)$ is $\sigma$-additive on $\Sigma_{2}$. In the case of a general Banach space $X$ the above discussion proves for every $x^{\prime} \in X^{\prime}$ the $\sigma$-additivity of the set function $F^{\prime} \mapsto\left\langle\int_{E} f d \beta\left(\cdot, F^{\prime}\right), x^{\prime}\right\rangle=\int_{E} f d\left(x^{\prime} \circ \beta\left(\cdot, F^{\prime}\right)\right)$, and so the assertion follows from Theorem 1 in $[6$, p. 318].

The following definition is inspired by $[15$, p. 482] and the definition in $[20$, p. 145] (see, however, Remark 7.3). 
Definttion 5.2. - The pair $(f, g)$ of functions $f: S_{1} \rightarrow \boldsymbol{K}$ and $g: S_{2} \rightarrow \boldsymbol{K}$ is said to be integrable with respect to the vector bimeasure: $\beta: \Sigma_{1} \times \Sigma_{2} \rightarrow X$ (or $\beta$-integrable for short) if the following three conditions hold:

(i) $f$ is $\beta(\cdot, F)$-integrable for all $F \in \Sigma_{2}$, and $g$ is $\beta(E, \cdot)$-integrable for all $E \in \Sigma_{1}$ (so that one obtains the vector measures ${ }_{f} \beta\left(S_{1}, \cdot\right): \Sigma_{2} \rightarrow X$ and $\beta_{g}\left(\cdot, S_{2}\right)$ : $\Sigma_{1} \rightarrow X$ defined by ${ }_{f} \beta\left(\delta_{1}, F^{\prime}\right)=\int_{S_{1}} f d \beta(\cdot, F), \beta_{g}\left(E, S_{2}\right)=\int_{S_{3}} g d \beta(E, \cdot)$, ef. Lemma 5.1);

(ii) $f$ is $\beta_{g}\left(\cdot, S_{2}\right)$-integrable and $g$ is ${ }_{f} \beta\left(S_{1}, \cdot\right)$-integrable;

(iii) $\int_{S_{1}} f d \beta_{s}\left(\cdot, S_{2}\right)=\int_{\mathcal{S}_{2}} g d_{f} \beta\left(S_{1}, \cdot\right)$.

If these conditions hold, each side of the equation in (iii) will be denoted by $\int(t, g) d \beta$.

Example 5.3. - This example shows that in the above definition (iii) does not follow from (i) and (ii). Choose $S_{1}=S_{2}=N=\{1,2,3, \ldots\}$, and let $\Sigma_{1}=\Sigma_{2}$ be the set of all subsets of $N$. Let $\left(a_{k}\right)$ be a sequence of positive numbers with $\sum_{k=1}^{\infty} a_{k}<\infty$
Construct inductively a function $f: B_{1} \rightarrow \boldsymbol{R}$ for which

$$
f(1) a_{1}=o_{1}>0, \quad f(k) a_{k}-f(k-1) a_{k-1}=c_{k}>0
$$

for $k>2$ and $\sum_{k=1}^{\infty} c_{k}<\infty$. Then choose a decreasing positive sequence $\left(b_{k}\right)$ such that $\sum_{k=1}^{\infty} f(k) a_{k} b_{k}<\infty$ (note that $\left.f(k)>0\right)$, and define the function $g: S_{2} \rightarrow \boldsymbol{R}$ by $g(n)=$ $=1 / b_{n}$. Define

$$
\beta(\{n\},\{n\})=a_{n} b_{n}, \quad \beta(\{n\},\{n+1\})=-a_{n} b_{n+1}
$$

for $n \in N$, and $\beta(\{m\},\{n\})=0$ if $m \neq n \neq m+1$. Since $\sum_{m=1}^{\infty} \sum_{n=1}^{\infty}|\beta(\{m\},\{n\})|<\infty$, it follows from well-known properties of summable families of numbers that the function $(E, F) \mapsto \beta(E, F)=\sum_{m \in E} \sum_{n \in F} \beta(\{m\},\{n\})$ on $\Sigma_{1} \times \Sigma_{2}$ is a bimeasure. We have

$$
\int_{S_{1}} f d \beta(\cdot,\{n\})=\sum_{m=1}^{\infty} f(m) \beta(\{m\},\{n\})=b_{n}\left(f(n) a_{n}-f(n-1) a_{n-1}\right)=b_{n} e_{n} \quad \text { if } n \geqslant 2
$$

and $\int_{\delta_{1}} f d \beta(\cdot,\{1\})=f(1) a_{1} b_{1}=b_{1} c_{1}$. Since

$$
\sum_{m=1}^{\infty} \sum_{n=1}^{\infty}|f(m) \beta(\{m\},\{n\})|=\sum_{m=1}^{\infty} f(m) a_{m}\left(b_{m}+b_{m+1}\right) \leqslant 2 \sum_{m=1}^{\infty} f(m) a_{m} b_{m}<\infty
$$

we can again use a property of summable families (i.e. the discrete version of 
Fubini's theorem) to show that for any $F \in \Sigma_{2}$,

$$
\sum_{n \in F} \sum_{m=1}^{\infty} f(m) \beta(\{m\},\{n\})=\sum_{n=1}^{\infty} f(m) \sum_{n \in F} \beta(\{m\},\{n\})
$$

(absolute convergence). Thus $f$ is $\beta(\cdot, F)$-integrable and

$$
\int_{S_{1}} f d \beta(\cdot, F)=\sum_{n \in F^{\prime}} \sum_{m=1}^{\infty} f(m) \beta(\{m\},\{n\})=\sum_{n \in F} b_{n} c_{n}
$$

Since

$$
\sum_{n=1}^{\infty} \sum_{m=1}^{\infty}|g(n) \beta(\{m\},\{n\})|=a_{1}+\sum_{n=2}^{\infty}\left(a_{n}+a_{n-1}\right)<\infty,
$$

we see similarly that $g$ is $\beta(E, \cdot)$-integrable for every $E \in \Sigma_{1}$, and

$$
\int_{S_{2}} g d \beta(E, \cdot)=\sum_{m \in E} \sum_{n=1}^{\infty} g(n) \beta(\{m\},\{n\})=\sum_{m \in E} a_{m}\left(g(m) b_{m}-g(m+1) b_{m+1}\right)=0
$$

for all $E \in \Sigma_{1}$.

Since

$$
\int_{S_{1}} f d \beta_{g}\left(\cdot, S_{2}\right)=0 \quad \text { and } \quad \int_{S_{2}} g d_{t} \beta\left(S_{1}, \cdot\right)=\sum_{n=1}^{\infty} g(n) b_{n} c_{n}=\sum_{n=1}^{\infty} e_{n}>0
$$

conditions (i) and (ii) of Definition 5.2 hold in this example, but (iii) does not.

The next result is an easy consequence of Theorem 8 in $[6, p .323]$ and Corollary 3.7.

Theorem 5.4. - (a) If $a_{j}, b_{j} \in \boldsymbol{K}$ for $j=1,2$, and if $f_{j}: S_{1} \rightarrow \boldsymbol{K}, g_{j}: S_{2} \rightarrow \boldsymbol{K}$ are functions such that the pairs $\left(f_{i}, g_{j}\right)$ for $i=1,2, j=1,2$, are $\beta$-integrable, then the pair $\left(a_{1} f_{1}+a_{2} f_{2}, b_{1} g_{1}+b_{2} g_{2}\right)$ is $\beta$-integrable, and

$$
\int\left(a_{1} f_{1}+a_{2} f_{2}, b_{1} g_{1}+b_{2} g_{2}\right) d \beta=\sum_{i=1}^{2} \sum_{j=1}^{2} a_{i} b_{j} \int\left(f_{i}, g_{j}\right) d \beta .
$$

(b) Let $Y$ be a Banach space and $T: X \rightarrow Y$ a bounded linear operator. Then $T \circ \beta$ is a vector bimeasure, and if the pair $(f, g)$ is $\beta$-integrable, it is To $\beta$-integrable, and $\int(f, g) d T \circ \beta=T\left(\int(f, g) d \beta\right)$.

LeMma 5.5. - Let $X=\boldsymbol{K}$, and let $f: S_{1} \rightarrow \boldsymbol{K}$ be $\beta\left(\cdot, F^{\prime}\right)$-integrable for every $F \in \Sigma_{2}$. Then $f$ is $\beta_{1}$-integrable (of. Theorem 4.4).

Proof. - We define $f \beta(E, F)=\int_{E} f d \beta(\cdot, F)$ for $E \in \Sigma_{1}, F \in \Sigma_{2}$. Then ${ }_{f} \beta$ is a bimeasure by Lemma 5.1 and the $\sigma$-additivity of the indefinite integral (cf. e.g. 
Theorem $20(a),(b)$ in $[6, p .114])$. Thus $\nu$ where $\nu(E)={ }_{f} \beta(E, \cdot), E \in \Sigma_{1}$, is a $c a\left(S_{2}, \Sigma_{2}\right)$-valued vector measure defined on $\Sigma_{1}$ (Theorem 4.4). We let $\chi_{F}^{\prime} \in c a\left(S_{2}, \Sigma_{2}\right)^{\prime}$ for $F \in \Sigma_{2}$ be as in the proof of Lemma 5.1 and write $D=\left\{\chi_{F}^{\prime}: F \in \Sigma_{2}\right\}$. Let $\left(E_{n}\right)$ be any decreasing sequence of members of $\Sigma_{1}$ such that $\bigcap_{n=1}^{\infty} E_{n}=\emptyset$. Then

$$
\sup _{F \in \Sigma_{\Sigma_{2}}} \int_{E_{n}}|f(s)| v\left(\chi_{F}^{\prime} \circ \beta_{1}, d s\right)=\sup _{E \in \Sigma_{2}} v\left(f \beta(\cdot, F), E_{n}\right)
$$

by Theorem $20(a)$ in [6, p. 114]. But $\sup _{F \in \Sigma_{2}} v\left({ }_{f} \beta(\cdot, F), E_{n}\right) \leqslant\|v\|\left(E_{n}\right) \rightarrow 0$ as $n \rightarrow \infty$ (Lemma 3.1.). Since $\sup _{F \in \Sigma_{2}}\left|\left\langle\lambda, \chi_{k}^{\prime}\right\rangle\right| \geqslant \frac{1}{4} v\left(\lambda, S_{2}\right)$ for all $\lambda \in c a\left(S_{2}, \Sigma_{2}\right)[6$, p. 97], Theorem 3.5 shows that $f$ is $\beta_{1}$-integrable.

The following theorem is analogous to part of the proposition in $[20, \mathbf{p} .145]$.

THeOREM 5.6. - For any function $f: S_{1} \rightarrow \boldsymbol{K}$ the following three conditions are equivalent:

(i) $f$ is $\beta(\cdot, F)$-integrable for all $F \in \Sigma_{2}$;

(ii) the pair $\left(f, \chi_{p}\right)$ is $\beta$-integrable for all $F \in \Sigma_{2}$;

(iii) the pair $(f, g)$ is $\beta$-integrable for every bounded $\Sigma_{2}$-measurable function $g: s_{2} \rightarrow K$.

Proof. - It is easily verified that (i) implies (ii). Assume now (ii). Let $g: S_{2} \rightarrow \boldsymbol{K}$ be bounded and $\Sigma_{2}$-measurable. Fix $x^{\prime} \in X^{\prime}$ and $E_{0} \in \Sigma_{1}$, and consider the bimeasure $x^{\prime} \beta=x^{\prime} \circ \beta$. By Theorem $8(f)$ in $[6, p .324]$ and Lemma 5.5, $f \chi_{E_{0}}$ is integrable with respect to the $c a\left(S_{2}, \Sigma_{2}\right)$-valued vector measure $x^{\prime} \beta_{1}\left(=\left(x^{\prime} \beta\right)_{1}\right)$. Let $g^{\prime} \in c a\left(S_{2}, \Sigma_{2}\right)^{\prime}$ be defined by $\left\langle g^{\prime}, \lambda\right\rangle=\int_{\delta_{3}} g d \lambda$. As $f \chi_{E_{0}}$ is $x^{\prime} \beta_{1}$-integrable, it is also integrable with respect to the scalar measure $g^{\prime} \circ x^{\prime} \beta_{1}$ (i.e. $\left.E \mapsto \int_{S_{2}} g d\left(x^{\prime} \circ \beta(E, \cdot)\right)=\left\langle x^{\prime}, \beta_{g}\left(E, S_{2}\right)\right\rangle\right)$.
We have

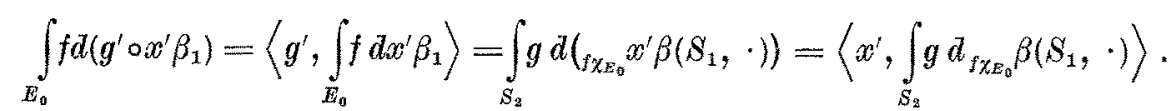

For any $E \in \Sigma_{1}$, we denote by ${ }_{f} \beta(E, \cdot)$ the vector measure $F \mapsto \int_{E} f d \beta(\cdot, F), F \in \Sigma_{2}$ (cf. Lemma 5.1), and define $\nu(E)=\int_{S_{2}} g d_{f} \beta(E, \cdot) \in X$ (cf. Theorem $8(c)$ in $[6$, p. 323]). Then we get by (1),

$$
\left\langle x^{\prime}, v(E)\right\rangle=\left\langle x^{\prime}, \int_{S_{2}} g d\left({ }_{f x_{E}} \beta\left(S_{1}, \cdot\right)\right)\right\rangle=\int_{E} f d\left(g^{\prime} \circ x^{\prime} \beta_{1}\right)=\int_{E} f d\left(x^{\prime} \circ \beta_{g}\left(\cdot, S_{2}\right)\right)
$$

for all $E \in \Sigma_{1}$. From Corollary 3.6 it thus follows that $f$ is $\beta_{g}\left(\cdot, S_{2}\right)$-integrable and $\int_{S_{1}} f d \beta_{g}\left(\cdot, S_{2}\right)=v\left(S_{1}\right)=\int_{S_{2}} g d_{f} \beta\left(S_{1}, \cdot\right)$, i.e. (iii) holds. As (iii) obviously implies (i), the theorem is proved. 
CoRoLlary 5.7. - If $f: \mathbb{S}_{1} \rightarrow \boldsymbol{K}$ is bounded and $\Sigma_{1}$-measurable, and $g: \mathbb{S}_{2} \rightarrow \boldsymbol{K}$ is bounded and $\Sigma_{2}$-measurable, then the pair $(f, g)$ is $\beta$-integrable, and

$$
\left|\int(f, g) d \beta\right| \leqslant\|\beta\|\left(S_{1}, S_{2}\right) \sup _{s \in S_{1}}|f(s)| \sup _{i \in E_{2}}|g(t)| \text {. }
$$

Proof. - The $\beta$-integrability of the pair $(f, g)$ follows at once from Theorem $8(c)$ in $[6, p .323]$ combined with the above theorem. (A direct elementary argument could also be given.) Clearly, we may assume that $f$ and $g$ are bounded in absolute value by 1 . Then

$$
\left|\int(f, g) d \beta\right|=\left|\int_{S_{1}} f d \beta_{g}\left(\cdot, S_{2}\right)\right| \leqslant\left\|\beta_{g}\left(\cdot, S_{2}\right)\right\|\left(S_{1}\right)
$$

by Theorem $8(c)$ in $[6, p .323]$. Let $\left(E_{j}\right)_{j=1}^{k}$ be a $\Sigma_{1}$-partition of $\$_{1}$, and $a_{j} \in K$, $\left|a_{j}\right| \leqslant 1, j=1, \ldots, k$. There is a sequence $\left(g_{n}\right)$ of $\Sigma_{2}$-simple functions bounded by 1 , converging uniformly to $g$. Since

$$
\left|\sum_{i=1}^{k} a_{j} \int_{S_{2}} g d \beta\left(E_{i}, \cdot\right)\right|=\lim _{n \rightarrow \infty}\left|\sum_{j=1}^{k} a_{j} \int_{S_{2}} g_{n} d \beta\left(E_{j}, \cdot\right)\right| \leqslant\|\beta\|\left(S_{1}, S_{2}\right)
$$

we have $\left\|\beta_{g}\left(\cdot, S_{2}\right)\right\| \leqslant\|\beta\|\left(S_{1}, S_{2}\right)$.

\section{6. - A Riesz type representation theorem.}

Throughout this section, $S_{1}$ and $S_{2}$ will be locally compact Hausdorff spaces. For $j=1,2, \mathfrak{B}_{j}$ will denote the Borel $\sigma$-algebra of $S_{j}$, i.e. $\mathfrak{B}_{i}$ is the $\sigma$-algebra generated by the open subsets of $S_{j}$. In the following discussion $S$ will stand for $S_{j}$ and $\mathscr{B}$ for $\mathfrak{B}_{j}$. An additive set function $\mu: \mathfrak{B} \rightarrow X$ is said to be regular, if for every $A \in \mathcal{B}$ and every $\varepsilon>0$ there exist a compact set $C$ and an open set $U$ such that $C \subset A \subset U$ and $|\mu(E)|<\varepsilon$ for all $E \in \mathfrak{B}, E \subset D \backslash C$. Let rea $(\mathcal{S}, \mathfrak{B}, X)$ denote the vector space of the regular vector measures $\mu: \mathcal{B} \rightarrow X$. We write simply $\operatorname{rca}(\mathbb{S}, \mathfrak{B}, \boldsymbol{K})=\operatorname{rea}(\mathbb{S}, \mathfrak{B})$.

According to the Riesz (-Markov-Kakutani) representation theorem the mapping $\mu \mapsto \varphi_{\mu}$ where for $\mu \in \operatorname{rca}(S, S) \varphi_{\mu} \in O_{0}(S)^{\prime}$ is defined by the formula $\int_{\bar{E}} f d \mu=$ $=\left\langle f, \varphi_{\mu}\right\rangle, f \in C_{0}(S)$, is an isometric isomorphism from the closed linear subspace $\operatorname{rea}(S, \mathscr{B})$ of $c a(S, \mathscr{B})$ onto $C_{0}(S)^{\prime}$ (cf. e.g. $[19$, p. 131]). To simplify notation we often identify $\mu$ and $\varphi_{\mu}$; we then use the common notation $M(S)$ for both of the spaces $\operatorname{rea}(S, \mathscr{B})$ and $C_{0}(S)^{\prime}$.

A vector measure $\mu: \mathfrak{B} \rightarrow X$ is known to be regular if (and obviously only if) $x^{\prime} \mu \in \operatorname{rca}\left(S, \mathscr{B}\right.$ ) for all $x^{\prime} \in X^{\prime}$ (cf. e.g. $[9$, p. 263] or [11, p. 159]). The proof of Corollary 2 in $[9$, p. 263] shows that for the regularity of $\mu$ it is in fact sufficient 
that $x^{\prime} \mu \in \operatorname{rca}(S, S)$ for all $x^{\prime}$ in some subset $D$ of $X^{\prime}$ which separates the points of $X$. The more elementary technique used in [11] would yield the regularity of $\mu$ in case $D \subset X_{1}^{\prime}$ is such that for some constant $C>0 \sup _{x^{\prime} \in D}\left|\left\langle x^{\prime}, x\right\rangle\right| \geqslant C|x|$ for all $x \in X$ and $x^{\prime} \mu \in \operatorname{rca}(S, \Re)$ for all $x^{\prime} \in D$. This generality will suffice for our purposes.

The next result can (essentially) be found e.g. in [6, p. 493]; clearly the proof given there also works in the case of a locally compact space $S$ and $C_{0}(S)$.

Lemma 6.1. - For $\mu \in \operatorname{rea}(S, \mathscr{B}, X)$ and $f \in O_{0}(S)$ write $T_{\mu} f=\int_{S} f d \mu$. Then $\mu \mapsto T_{\mu}$ is a linear bijection from $\operatorname{rea}(S, \mathfrak{B}, X)$ onto the set of all weakly compact linear operators $T: C_{0}(S) \rightarrow X$. Moreover, $\left|T_{\mu}\right|=\|\mu\|(S)$ for all $\mu \in \operatorname{rea}(S, \mathfrak{B}, X)$.

REMARK 6.2. - We shall always regard $X$ canonically as a closed linear subspace of $X^{\prime \prime}$. Let $T: O_{0}(S) \rightarrow X$ be a bounded linear operator and $T^{\prime \prime}: M(S) \rightarrow X^{\prime \prime}$ its second adjoint. For $E \in \mathfrak{B}$ we write $\mu(E)=T^{\prime \prime} \chi_{\mathbb{Z}}^{\prime} \in X^{\prime \prime}$ where $\chi_{B}^{\prime} \in M(S)^{\prime}$ is defined by $\left\langle\chi_{E}^{\prime}, \lambda\right\rangle=\lambda(E), \lambda \in M(S)$. Then $T$ is weakly compact if and only if $\mu(E) \in X$ for all $E \in \mathscr{B}$, and in this case $\mu \in r c a(S, \mathfrak{B}, X)$ and $T=T_{\mu}[6, p .493]$. It was observed in $[3$, p. 154$]$ that the $\sigma$-additivity of the set function $\mu: \mathscr{B} \rightarrow X^{n}$ already implies the weak compactness of $T$. Here is a proof of this fact. Since $\lim _{n \rightarrow \infty} \mu\left(E_{n}\right)=0$, i.e. $T^{\prime} x^{\prime}\left(E_{n}\right)=\left\langle\mu\left(E_{n}\right), x^{\prime}\right\rangle \rightarrow 0$ uniformly in $x^{\prime} \in X_{1}^{\prime}$, for any decreasing sequence $\left(E_{n}\right)$ of members of $\mathfrak{B}$ satisfying $\bigcap_{n=1}^{\infty} F_{n}=\emptyset, T^{\prime}$ is weakly compact (see Theorem 1 in $[6$, p. 305]), and so is $T[6, \mathrm{p} .485]$.

We intend to establish in the context of vector bimeasures an analogue of Lemma 6.1. First we need to consider extensions of certain bilinear operators.

LEMma 6.3. - Let $B: C_{0}\left(S_{1}\right) \times C_{0}\left(S_{2}\right) \rightarrow X$ be a bounded bilinear operator. There is a unique bilinear operator $B_{e}: C_{0}\left(S_{1}\right)^{\prime \prime} \times C_{0}\left(S_{2}\right)^{\prime \prime} \rightarrow X^{\prime \prime}$ which is an extension of $B$ (when each of the Banach spaces $C_{0}\left(S_{1}\right), C_{0}\left(S_{2}\right)$ and $X$ is canonically embedded in its bidual) and separately continuous when each of the spaces $O_{0}\left(S_{1}\right)^{\prime \prime}, O_{0}\left(S_{2}\right)^{\prime \prime}$ and $X^{\prime \prime}$ is equipped with its weak* topology. The norms of $B$ and $B_{e}$ are the same, and $B_{e}$ depends linearly on $B$.

Proof. - According to a well-known result due to Grothendieck [8], every bounded linear operator from $C_{0}\left(S_{1}\right)$ to $C_{0}\left(S_{2}\right)^{\prime}$ is weakly compact. (One way of seeing this is to combine Theorem 6 in $[6$, p. 494] with Theorem 4 in $[6$, p. 308] and Proposition 5.1 in $\left[20\right.$, p. 135].) Thus, for each $x^{\prime} \in X^{\prime}, x^{\prime} \circ B$ has a unique separately weak* continuous extension $B_{e}^{x^{\prime}}: O_{0}\left(S_{1}\right)^{\prime \prime} \times O_{0}\left(\mathcal{A}_{2}\right)^{\prime \prime} \rightarrow \boldsymbol{K}$, and $\left|B_{e}^{a^{\prime}}\right|=\left|x^{\prime} \circ B\right|$ (see e.g. $\left[21\right.$, p. 365]). We define $\left(B_{e}(u, v)\right)\left(x^{\prime}\right)=B_{e}^{x^{\prime}}(u, v)$ for $u \in C_{0}\left(S_{1}\right)^{\prime \prime}, v \in C_{0}\left(S_{2}\right)^{\prime \prime}, x^{\prime} \in X^{\prime}$. Then $B_{e}(u, v) \in X^{\prime \prime}$. In fact, $B_{e}^{a x^{\prime}+b y^{\prime}}=a B_{e}^{x^{\prime}}+b B_{e}^{y^{\prime}}$ for $x^{\prime}, y^{\prime} \in X^{\prime}, a, b \in \boldsymbol{K}$, because $a B_{e}^{x^{\prime}}+b B_{e}^{y^{\prime}}$ is separately weak* continuous and extends $\left(a x^{\prime}+b y^{\prime}\right) \circ B$. Thus $B_{e}(u, v)$ : $X^{\prime} \rightarrow \boldsymbol{K}$ is linear. Since $\left|\left(B_{e}(u, v)\right)\left(x^{\prime}\right)\right|=\left|B_{e}^{x^{\prime}}(u, v)\right| \leqslant\left|x^{\prime}\right||B||u||v|, B_{e}(u, v)$ is continuous. This also shows that $\left|B_{e}\right|<|B|$, so that $\left|B_{e}\right|=|B|$. The separate weak*-to- 
weak* continuity of $B_{\theta}$ is obvious, and so is its uniqueness, because $C_{0}\left(\mathcal{S}_{j}\right)$ is weak* dense in $O_{0}\left(S_{j}\right)^{\prime \prime}$. Finally, if $B_{1}, B_{2}: O_{0}\left(S_{1}\right) \times O_{0}\left(S_{2}\right) \rightarrow X$ are bilinear and bounded, and $a, b \in \boldsymbol{K}, a\left(B_{1}\right)_{e}+b\left(B_{2}\right)_{e}$ is a separately weak*-to-weak* continuous extension of $a B_{1}+b B_{2}$, and so $a\left(B_{1}\right)_{e}+b\left(B_{2}\right)_{e}=\left(a B_{1}+b B_{2}\right)_{e}$.

Definition 6.4. - If $B$ and $B_{\theta}$ are as in the preceding lemma, $B_{e}$ is called the canonical extension of $B$.

We shall make use of the canonical extension in proving representation theorems for bounded bilinear operators from $O_{0}\left(S_{1}\right) \times O_{0}\left(S_{2}\right)$ to $X$. As a preliminary step, let us treat the scalar case.

Notation. - If $f: S_{j} \rightarrow \boldsymbol{K}$ is a bounded Borel (i.e., $\mathfrak{B}_{j}$-measurable) function, $f^{\prime} \in M\left(S_{3}\right)^{\prime}$ will denote the functional defined by $\left\langle f^{\prime}, \lambda\right\rangle \underset{s_{j}}{=} f d \lambda, \lambda \in M\left(S_{j}\right)$.

LEMMA 6.5. - If $b: C_{0}\left(S_{1}\right) \times C_{0}\left(S_{2}\right) \rightarrow \boldsymbol{K}$ is a bounded bilinear form, there is a unique function $\beta_{b}: \mathfrak{B}_{1} \times \mathcal{B}_{2} \rightarrow \boldsymbol{K}$ satisfying the following two conditions:

(i) $\beta_{b}(E, \cdot) \in \operatorname{rea}\left(S_{2}, \mathscr{B}_{2}\right)$ for all $E \in \Re_{1}$ and $\beta_{b}(\cdot, F) \in \operatorname{rca}\left(S_{1}, \mathfrak{B}_{1}\right)$ for all $F \in \mathscr{B}_{2}$;

(ii) $\int(h, k) d \beta_{b}=b(h, k)$ for all $h \in O_{0}\left(S_{1}\right), k \in O_{0}\left(S_{2}\right)$.

If $b_{e}: M\left(S_{1}\right)^{\prime} \times M\left(S_{2}\right)^{\prime} \rightarrow \boldsymbol{K}$ is the canonical extension of $b$ and $f: S_{1} \rightarrow \boldsymbol{K}, g: S_{2} \rightarrow \boldsymbol{K}$ are bounded Borel functions, we have $b_{e}\left(f^{\prime}, g^{\prime}\right)=\left[(f, g) d \beta_{b}\right.$. The norm of $b$ equals $\left\|\beta_{b}\right\|\left(S_{1}, S_{2}\right)$. Conversely, if $\beta: \mathfrak{B}_{1} \times \mathscr{B}_{2} \rightarrow \boldsymbol{K}$ satisfies (i), there is a unique bounded bilinear form $b: C_{0}\left(S_{1}\right) \times C_{0}\left(\mathcal{S}_{2}\right) \rightarrow \boldsymbol{K}$ such that $\beta=\beta_{b}$.

Proof. - We define $\beta_{b}$ by the formula $\beta_{b}(E, F)=b_{e}\left(\chi_{E}^{\prime}, \chi_{F}^{\prime}\right), E \in \mathfrak{B}_{1}, F \in \mathfrak{B}_{2}$. From the separate weak* continuity of $b_{\theta}$ it follows that e.g. for all bounded Borel functions $f: S_{1} \rightarrow \boldsymbol{K}$ the functional $b_{e}\left(f^{\prime}, \cdot\right): M\left(S_{2}\right)^{\prime} \rightarrow \boldsymbol{K}$ is the canonical image of some $\lambda_{f} \in M\left(S_{2}\right)\left[6\right.$, p. 421]. In particular, (i) holds. Let $f: \$_{1} \rightarrow K$ and $g: \$_{2} \rightarrow K$ be bounded Borel functions. The pair $(f, g)$ is $\beta_{b}$-integrable by Corollary 5.7. The measure $F \mapsto \int_{S_{1}} f d \beta_{b}(\cdot, F), F \in \mathfrak{B}_{2}$, is just $\lambda_{f}$ (as can be seen by approximating $f$ uniformly by $\mathfrak{B}_{1}$-simple functions). By definition we thus have $\int(f, g) d \beta_{b}=\int_{S_{2}} g d \lambda_{f}=$ $=b_{e}\left(f^{\prime}, g^{\prime}\right)$. In particular, (ii) holds. We now show that if any $\beta: \mathfrak{B}_{1} \times \mathfrak{B}_{2} \rightarrow \boldsymbol{K}$ in place of $\beta_{b}$ satisfies (i) and (ii), then $\|\beta\|\left(B_{1}, S_{2}\right)=|b|$; this will also prove (by an obvious linearity argument) the uniqueness of $\beta_{b}$. Consider the vector measure $\beta_{1}: \mathfrak{B}_{1} \rightarrow c a\left(S_{2}, \mathfrak{B}_{2}\right)$ defined by $\left(\beta_{1}(E)\right)\left(F^{\prime}\right)=\beta(E, F)$ (cf. Theorem 4.4). The values of $\beta_{1}$ lie in $r a\left(S_{2}, \Re_{2}\right)$, and it is regular (see the discussion preceding Lemma 6.1, and $\left[6\right.$, p. 97]). Therefore, $\left\|\beta_{1}\right\|\left(S_{1}\right)=\sup \left\{\left|\int_{\beta_{1}} h d \beta_{1}\right|: h \in C_{0}\left(S_{1}\right),|h| \leqslant 1\right\}$ by Lemma 6.1.
Since

$$
\int_{S_{1}} h d \beta_{1} \in \operatorname{rea}\left(S_{2}, \mathfrak{B}_{2}\right) \quad \text { for all } h \in C_{0}\left(S_{1}\right)
$$


we have

$$
\left|\int_{S_{1}} h d \beta_{1}\right|=\sup \left\{\left|\int_{S_{2}} k d\left(\int_{S_{1}} h d \beta_{1}\right)\right|: k \in C_{0}\left(S_{2}\right),|k| \leqslant 1\right\}=\sup _{|k| \leqslant 1}|b(h, k)|,
$$

since

$$
\int_{S_{\mathrm{z}}} k d\left(\int_{S_{1}} h d \beta_{1}\right)=\int(h, k) d \beta=b(h, k) .
$$

Thus

$$
\|\beta\|\left(S_{1}, S_{2}\right)=\left\|\beta_{1}\right\|\left(S_{1}\right)=\sup _{|h| \leqslant 1} \sup _{|k| \leqslant 1}|b(h, k)|=|b|
$$

by Theorem 4.4. As to the last assertion, observe that $(h, k) \mapsto \int(h, k) d \beta$ is a bounded bilinear form on $C_{0}\left(S_{1}\right) \times C_{0}\left(S_{2}\right)$ (cf. Theorem 4.5 and Corollary 5.7).

THeOREM 6.6. - Let $B: C_{0}\left(S_{1}\right) \times C_{0}\left(S_{2}\right) \rightarrow X$ be a bounded bilinear operator. There exists a unique mapping $\beta_{B}: \aleph_{1} \times \aleph_{2} \rightarrow X^{\prime \prime}$ satisfying the following two conditions:

(i) if $x^{\prime} \in X^{\prime}$, then the function $F \mapsto\left\langle x^{\prime}, \beta_{B}(E, F)\right\rangle$ is in $r c a\left(S_{2}, \mathscr{B}_{2}\right)$ for all $E \in \mathfrak{B}_{1}$, and the function $E \mapsto\left\langle x^{\prime}, \beta_{B}(E, F)\right\rangle$ is in $\operatorname{rea}\left(S_{1}, \mathfrak{B}_{1}\right)$ for all $F \in \mathfrak{B}_{2}$;

(ii) for all $x^{\prime} \in X^{\prime}, h \in C_{0}\left(S_{1}\right)$ and $k \in C_{0}\left(S_{2}\right),\left\langle B(h, k), x^{\prime}\right\rangle$ equals the integral of the pair $(h, k)$ with respect to the bimeasure $(E, F) \mapsto\left\langle x^{\prime}, \beta_{B}(E, F)\right\rangle, \quad E \in \mathfrak{B}_{1}$, $F \in \mathscr{S}_{2}$. We have

$$
\beta_{B}(E, F)=B_{e}\left(\chi_{E}^{\prime}, \chi_{F}^{\prime}\right) \quad \text { for } E \in \mathfrak{B}_{1}, F \in \mathfrak{B}_{2},
$$

and $\left\|\beta_{B}\right\|\left(\mathbb{S}_{1}, \mathbb{S}_{2}\right)=|B|$.

Proof. - Define $\beta_{B}$ by (1). As for any $x^{\prime} \in X^{\prime}$ the canonical extension of $x^{\prime} \circ B$ is $x^{\prime} \circ\left(B_{e}\right)$ (with the interpretation $\left.x^{\prime} \in X^{\prime \prime \prime}\right)$, (i), (ii) and the uniqueness statement follow from Lemma 6.5. Since $\left|\alpha^{\prime} \circ B\right|=\left\|\alpha^{\prime} \circ \beta_{B}\right\|\left(B_{1}, S_{2}\right)$ (Lemma 6.5), $|B|=\|\beta\|$. $\cdot\left(\$_{1}, S_{2}\right)$ by Lemma 4.3 .

We are going to characterize those bounded bilinear operators $B: C_{0}\left(S_{1}\right) \times$ $\times C_{0}\left(\mathscr{S}_{2}\right) \rightarrow X$ for which $\beta_{B}\left(\mathscr{B}_{1} \times \mathscr{B}_{2}\right) \subset X$. Let us prepare the proof of that result with a lemma.

LEMMA 6.7. - Let $B: C_{0}\left(S_{1}\right) \times C_{0}\left(S_{2}\right) \rightarrow X$ be a bounded bilinear operator and $B_{e}$ its canonical extension. Let $\varphi \in C_{0}\left(S_{1}\right)^{\prime \prime}$ be such that $B_{e}\left(\varphi, k^{\prime}\right) \in X$ for all $k \in C_{0}\left(S_{2}\right)$. Then $B_{e}(\varphi, \cdot): C_{0}\left(S_{2}\right)^{\prime \prime} \rightarrow X^{\prime \prime}$ is the second adjoint of the operator $k \mapsto B_{e}\left(\varphi, k^{\prime}\right)$ from $C_{0}\left(S_{2}\right)$ to $X$.

Proof. - Both $B_{e}(\varphi, \cdot)$ and the second adjoint of $k \mapsto B_{e}\left(\varphi, k^{\prime}\right)$ are continuous from $\sigma\left(C_{0}\left(S_{2}\right)^{\prime \prime}, C_{0}\left(S_{2}\right)^{\prime}\right)$ to $\sigma\left(X^{\prime \prime}, X^{\prime}\right)$. As they agree on the canonical image of $C_{0}\left(S_{2}\right)$, which is weak* dense in $C_{0}\left(S_{2}\right)^{\prime \prime}$, they are the same.

9 - Annali di Matematica 
Theoren 6.8. - Let $B: C_{0}\left(S_{1}\right) \times C_{0}\left(S_{2}\right) \rightarrow X$ and $\beta_{B}: \Re_{1} \times \Re_{2} \rightarrow X^{\prime \prime}$ be as in Theorem 6.6. The following six conditions are equivalent:

(i) $\beta_{B}(E, \cdot)$ and $\beta_{B}(\cdot, F)$ are regular for all $E \in \mathscr{B}_{\mathbf{1}}, F \in \mathfrak{B}_{2}$;

(ii) $\beta_{B}(E, \cdot)$ and $\beta_{B}(\cdot, F)$ are $\sigma$-additive for all $E \in \mathfrak{B}_{1}, F \in \mathfrak{B}_{2}$;

(iii) $\beta_{B}(E, F) \in X$ for all $E \in \Re_{1}, F \in \Re_{2}$;

(iv) $B_{e}\left(f^{\prime}, g^{\prime}\right) \in X$ for all bounded Borel functions $f: S_{1} \rightarrow \boldsymbol{K}, g: S_{2} \rightarrow \boldsymbol{K}$;

(v) $B_{e}\left(f^{\prime}, g^{\prime}\right) \in X$ whenever $f \in C_{0}\left(S_{1}\right) \cup\left\{\chi_{z}: E \subset S_{1}\right.$ is open $\}$ and $g \in C_{0}\left(S_{2}\right) \cup$ $\cup\left\{\chi_{F}: F \subset S_{2}\right.$ is open $\}$;

(vi) $B_{\theta}\left(C_{0}\left(S_{1}\right)^{\prime \prime} \times C_{0}\left(S_{2}\right)^{\prime \prime}\right) \subset X$.

PRoof. - Since every additive and regular $\mu: \mathfrak{B}_{j} \rightarrow X^{\prime \prime}$ is $\sigma$-additive (see e.g. the proof of Theorem 13 in $[6$, p. 138], or $[4$, p. 510]), (i) implies (ii). Assume (ii). For each $h \in C_{0}\left(S_{1}\right)$ there is a sequence $\left(f_{n}\right)$ of $\mathfrak{S}_{1}$-simple functions converging uniformly to $h$. Each function $F \mapsto B_{e}\left(f_{n}^{\prime}, \chi_{F}^{\prime}\right)$ is $\sigma$-additive, and the sequence of these functions converges uniformly on $\mathfrak{B}_{2}$ to the function $F \mapsto B_{e}\left(h^{\prime}, \chi_{F}^{\prime}\right)$, which is therefore $\sigma$-additive, too. But Lemma 6.7 shows that $B_{e}\left(h^{\prime}, \chi_{F}^{\prime}\right)=B(h, \cdot)^{\prime \prime}\left(\chi_{F}^{\prime}\right)$, and so $B_{\varepsilon}\left(h^{\prime}, \chi_{F}^{\prime}\right) \in X$ for all $F \in \mathfrak{B}_{2}$ (see Remark 6.2). Using Lemma 6.7 again we see that if $F \in \mathscr{B}_{2}$, the second adjoint of the operator $h \mapsto B_{e}\left(h^{\prime}, \chi_{F}^{\prime}\right), h \in C_{0}\left(S_{1}\right)$, has the value $B_{e}\left(\chi_{E}^{\prime}, \chi_{F}^{\prime}\right)$ for all $E \in \Re_{1}$. Since $E \mapsto B_{e}\left(\chi_{E}^{\prime}, \chi_{F}^{\prime}\right)$ is $\sigma$-additive, (iii) holds (Remark 6.2). As each bounded Borel function on $S_{j}$ can be approximated uniformly by $\mathfrak{B}_{j}$-simple functions, (iii) implies (iv). Clearly, (iv) implies (v). Assume now (v). To show that then (vi) holds, we shall use a result of Grothendieck which says that a bounded linear operator $T: C_{0}\left(S_{j}\right) \rightarrow X$ is weakly compact if (and only if) $T^{\prime \prime}\left(\chi_{E}\right) \subset X$ for all open sets $E \subset S_{j}$ (see [8, pp.160-161]). For a fixed function $h \in C_{0}\left(S_{1}\right), B_{e}\left(h^{\prime}, \cdot\right): M\left(S_{2}\right)^{\prime} \rightarrow X^{\prime \prime}$ is the second adjoint of $B(h, \cdot)$ (Lemma 6.7$)$. Since $B_{e}\left(h^{\prime}, \chi_{F^{\prime}}^{\prime}\right) \in X$ for all open sets $F \subset S_{2}, B(h, \cdot)$ is weakly compact, and so $B_{e}\left(h^{\prime}, \psi\right) \in X$ for all $\psi \in M\left(S_{2}\right)^{\prime}\left[6\right.$, p. 482]. For a fixed functional $\psi \in M\left(S_{2}\right)^{\prime}$, the operator $h \mapsto B_{e}$. $\cdot\left(h^{\prime}, \psi\right)$ from $C_{0}\left(S_{1}\right)$ to $X$ has $B_{e}(\cdot, \psi): M\left(S_{1}\right)^{\prime} \rightarrow X$ as its second adjoint (Lemma 6.7). If $g: S_{2} \rightarrow \boldsymbol{K}$ is in $C_{0}\left(S_{k}\right)$ or if $g$ is the characteristic function of an open set, $B_{e}\left(\chi_{E}^{\prime}, g^{\prime}\right) \in X$ for every open set $E \subset S_{1}$, so that the operator $h \mapsto B_{e}\left(h^{\prime}, g^{\prime}\right)$ is wealkly compact by Grothendieck's theorem. Thus $B_{e}\left(\varphi, g^{\prime}\right) \in X$ for all $\varphi \in M\left(S_{1}\right)^{\prime}[6, p .482]$. Fix now $\varphi \in M\left(S_{1}\right)^{2}$. By Lemma $6.7, B_{e}(\varphi, \cdot)$ is the second adjoint of the operator $k \mapsto B_{e}\left(\varphi, k^{\prime}\right)$ from $C_{0}\left(S_{2}\right)$ to $X$, and since $B_{e}\left(\varphi, \chi_{F}^{\prime}\right) \in X$ for ail open sets $F \subset S_{2}$, the latter operator is weakly compact by Grothendieck's theorem, and so $B_{e}(\varphi, \psi) \in X$ for all $\psi \in M\left(S_{2}\right)^{\prime}$, i.e., (vi) holds. Clearly, (vi) implies (iii). In view of Theorem 6.6 and the Orlicz-Pettis theorem [6, p. 318], (iii) implies (ii). Theorem 6.6 combined with the discussion preceding Lemma 6.1 shows that (ii) implies (i).

We are now ready to prove our main representation theorem. A vector bimeasure $\beta: \mathfrak{B}_{1} \times \mathscr{B}_{2} \rightarrow X$ is said to be separately regular if $\beta(E, \cdot) \in \operatorname{rca}\left(S_{2}, \mathscr{B}_{2}, X\right)$ for each $E \in \mathfrak{B}_{1}$ and $\beta\left(\cdot, F^{\prime}\right) \in \operatorname{rca}\left(\mathcal{S}_{1}, \mathfrak{B}_{1}, X\right)$ for all $F \in \mathscr{B}_{2}$. We denote by $\operatorname{srca}\left(\mathcal{S}_{1}, \mathfrak{B}_{1}\right.$; $S_{2}, \mathfrak{B}_{2} ; X$ ) the set of the separately regular vector bimeasures $\beta: \mathfrak{B}_{1} \times \mathfrak{M}_{2} \rightarrow X$. It is 
easy to verify that $\operatorname{srca}\left(\mathscr{S}_{1}, \mathscr{B}_{1} ; \mathbb{S}_{2}, \mathscr{B}_{2} ; X\right)$ is a closed linear subspace of the Banach space of all vector bimeasures $\beta: \mathscr{B}_{1} \times \mathscr{B}_{2} \rightarrow X$ equipped with the semivariation norm (Section 4).

THeorem 6.9. - Denote $B_{\beta}(h, k)=\int(h, k) d \beta$ for all $\beta \in \operatorname{srca}\left(S_{1}, \mathfrak{B}_{1} ; S_{2}, \mathfrak{B}_{2} ; X\right)$ and $h \in O_{0}\left(S_{1}\right), k \in C_{0}\left(S_{2}\right)$. Then the mapping $\beta \mapsto B_{\beta}$ is an isometric linear bijection from srea $\left(S_{1}, \Re_{1} ; S_{2}, \Re_{2} ; X\right)$ onto the Banach space of those bounded bilinear operators $B: C_{0}\left(S_{1}\right) \times C_{0}\left(S_{2}\right) \rightarrow X$ for which $B_{e}\left(C_{0}\left(S_{1}\right)^{\prime \prime} \times C_{0}\left(S_{2}\right)^{\prime \prime}\right) \subset X$. Moreover, $\left(B_{\beta}\right)_{e}\left(f^{\prime}, g^{\prime}\right)=$ $=\int(f, g) d \beta$ for all bounded Borel funetions $f: S_{1} \rightarrow \boldsymbol{K}, g: S_{2} \rightarrow \boldsymbol{K}$ and $\beta \in \operatorname{srca}\left(S_{1}, \mathscr{B}_{1}\right.$; $\left.\mathcal{S}_{2}, \mathfrak{B}_{2} ; X\right)$. In particular, $\left(B_{\beta}\right)_{e}\left(\chi_{E}^{\prime}, \chi_{F}^{\prime}\right)=\beta(E, F)$ for $E \in \mathfrak{M}_{1}, F \in \mathscr{M}_{2}$.

Proof. - Suppose $\beta \in \operatorname{srca}\left(S_{1}, \mathscr{B}_{1} ; S_{2}, \mathscr{B}_{2} ; X\right)$. Then $B_{\beta}: C_{0}\left(S_{1}\right) \times C_{0}\left(S_{2}\right) \rightarrow X$ is a bounded bilinear operator by Theorem 5.4 (or a direct elementary argument), Corollary 5.7 and Theorem 4.5. Let $f: S_{1} \rightarrow \boldsymbol{K}$ and $g: S_{2} \rightarrow \boldsymbol{K}$ be bounded Borel functions. If $x^{\prime} \in X^{\prime},\left(x^{\prime} \circ B_{\beta}\right)_{e}\left(f^{\prime}, g^{\prime}\right)=\int(f, g) d\left(x^{\prime} \circ \beta\right)$ by Lemma 6.5, because $x^{\prime} \circ B_{\beta}$. $\cdot(h, k)=\int(h, k) d\left\langle x^{\prime} \circ \beta\right)$ for $h \in C_{0}\left(S_{1}\right), k \in C_{0}\left(S_{2}\right)$ (Theorem 5.4 $\left.(b)\right)$. As $(u, v) \mapsto$ $\mapsto\left\langle x^{\prime},\left(B_{\beta}\right)_{\theta}(u, v)\right\rangle$ is the canonical extension of $x^{\prime} \circ B_{\beta}$, it follows that

$$
\left\langle x^{\prime},\left(B_{\beta}\right)_{e}\left(f^{\prime}, g^{\prime}\right)\right\rangle=\left(x^{\prime} \circ B_{\beta}\right)_{e}\left(f^{\prime}, g^{\prime}\right)=\left\langle x^{\prime}, \int(f, g) d \beta\right\rangle
$$

(Theorem 5.4 $(b))$, i.e. $\left(B_{\beta}\right)_{e}\left(f^{\prime}, g^{\prime}\right)=\int(f, g) d \beta \quad(\in X)$. Thus Theorem 6.8 shows that $\left(B_{\beta}\right)_{\epsilon}\left(O_{0}\left(S_{1}\right)^{\prime \prime} \times O_{0}\left(S_{2}\right)^{\prime \prime}\right) \subset X$. It is easily verified that the mapping $\beta \mapsto B_{\beta}, \beta \in$ srea . $\cdot\left(S_{1}, \mathfrak{B}_{1} ; S_{2}, \mathfrak{B}_{2} ; X\right)$, is linear. Since $\left(B_{\beta}\right)_{e}\left(\chi_{E}^{\prime}, \chi_{F}^{\prime}\right)=\beta(E, F)$ for all $E \in \mathfrak{B}_{1}, F^{\prime} \in \mathscr{B}_{2}$, it is injective. Let now $B: C_{0}\left(S_{1}\right) \times C_{0}\left(S_{2}\right) \rightarrow X$ be any bounded bilinear operator satisfying the six equivalent conditions in Theorem 6.8. If $\beta=\beta_{B}: \mathfrak{B}_{1} \times \mathscr{B}_{2} \rightarrow X \subset X^{\prime \prime}$ is defined as in Theorem 6.6 (so that $\beta \in \operatorname{srca}\left(S_{1}, \mathfrak{B}_{1} ; S_{2}, \mathfrak{B}_{2} ; X\right)$ by Theorem 6.8), then by Theorem $6.6 B=B_{\beta}$ (because $\left\langle x^{\prime}, \int(h, k) d \beta\right\rangle=\int(h, k) d\left(x^{\prime} \circ \beta\right)=\left\langle x^{\prime}, B(h, k)\right\rangle$, $\left.x^{\prime} \in X, h \in C_{0}\left(S_{1}\right), k \in C_{0}\left(S_{2}\right)\right)$, and $\|\beta\|\left(S_{1}, S_{2}\right)=|B|$.

REMARK 6.10. - If $B: O_{0}\left(S_{1}\right) \times O_{0}\left(S_{2}\right) \rightarrow X$ is a bounded bilinear operator such that $B_{e}\left(C_{0}\left(S_{1}\right)^{\prime \prime} \times O_{0}\left(S_{2}\right)^{\prime \prime}\right) \subset X$, it is clear from Lemma 6.7 that $B(\cdot, k): C_{0}\left(S_{1}\right) \rightarrow X$ and $B(h, \cdot): C_{0}\left(S_{2}\right) \rightarrow X$ are weakly compact operators for all $k \in C_{0}\left(B_{2}\right), h \in C_{0}\left(S_{1}\right)$ (see $[6, p .482]$ ). The weak compactness of all these operators does not in turn, however, imply that $B_{e}\left(O_{0}\left(S_{1}\right)^{\prime \prime} \times O_{0}\left(S_{2}\right)^{\prime \prime}\right) \subset X$. For example, denote as usual $c_{0}=C_{0}(N)$ and define $B: c_{0} \times c_{0} \rightarrow c_{0}$ by pointwise multiplication. It is clear that when $l^{\infty}$, the set of all bounded sequences, is in the usual way identified with $c_{0}^{\prime \prime}$, $B_{e}(f, g)=f g$ for all $f, g \in l^{\infty}$. In fact, the bilinear operator $(f, g) \mapsto f g$ extends $B$ and is obviously separately weak*-to-weak* continuous. Thus $B_{e}\left(c_{0}^{\prime \prime} \times c_{0}^{\prime \prime}\right)=l^{\infty}$, although all the operators $B(\cdot, k): c_{0} \rightarrow c_{0}$ and $B(h, \cdot): c_{0} \rightarrow c_{0}$ for $h, k \in c_{0}$ are even compact.

We conclude this section with a theorem which one would expect to be true of a satisfactory bilinear analogue of a weakly compact operator. We first prove a lemma. 
LEMMA 6.11. - Let $\beta \in \operatorname{srca}\left(\mathscr{S}_{1}, \mathscr{B}_{1} ; S_{2}, \mathscr{B}_{2} ; X\right)$ and let $B=B_{\beta}: C_{0}\left(S_{1}\right) \times C_{0}\left(S_{2}\right) \rightarrow X$ be defined as in Theorem 6.9. Then the sets $U=\left\{\beta(E, F): E \in \mathfrak{B}_{1}, F \in \mathfrak{B}_{2}\right\}$ and $V=\left\{B_{\beta}(h, k): h \in C_{0}\left(S_{1}\right), k \in C_{0}\left(S_{2}\right)\right\}$ span the same closed linear subspace of $X$.

Proof. - Let $Y_{1}$ be the closed linear hull of $U$ and $Y_{2}$ that of $V$. Fix $k \in S_{0}\left(S_{2}\right)$. We have $B_{e}\left(\chi_{E}^{\prime}, k^{\prime}\right)=B(\cdot, k)^{\prime \prime}\left(\chi_{E}^{\prime}\right)$ for all $E \in \Re_{1}$ by Lemma 6.7. As $(B \cdot, k)^{\prime \prime}$ : $C_{0}\left(S_{1}\right)^{n} \rightarrow X$ is continuous from $\sigma\left(O_{0}\left(S_{1}\right)^{\prime \prime}, C_{0}\left(S_{2}\right)^{\prime}\right)$ to $\sigma\left(X, X^{\prime}\right)$ (note that $B(\cdot, k)$ is weakly compact), and $C_{0}\left(S_{1}\right)$ is weak* dense in $O_{0}\left(S_{1}\right)^{\prime \prime}, B_{e}\left(\chi_{E}^{\prime}, k^{\prime}\right) \in Y_{2}$ for all $E \in \mathfrak{B}_{1}$, because $Y_{2}$ is $\sigma\left(X, X^{\prime}\right)$-closed $\left[6\right.$, p. 422]. Fix now $E \in \mathfrak{B}_{1}$. By Lemma 6.7 again, $B_{e}\left(\chi_{E}^{\prime}, \cdot\right)$ is the second adjoint of the weakly compact $\left[6\right.$, p. 482] operator $k \mapsto B_{e}$. $\cdot\left(\chi_{E}^{\prime}, k^{\prime}\right)$, and so a similar reasoning shows that $B_{e}\left(\chi_{E}^{\prime}, \chi_{F}^{\prime}\right) \in Y_{2}$ for all $F \in \Re_{2}$. Thus $Y_{1} \subset Y_{2}$. Conversely, it follows from Definitions 5.2 and 3.3 that each $x \in V$ can be approximated in norm by linear combinations of elements from $U$.

REMaRK 6.12. - The method used in the first part of the above proof also shows that if $B: C_{0}\left(S_{1}\right) \times C_{0}\left(S_{2}\right) \rightarrow X$ is a bounded bilinear operator whose range is contained in a closed linear subspace $X$ of $X$, and such that $B_{\varepsilon}\left(C_{0}\left(S_{1}\right)^{\prime \prime} \times C_{0}\left(S_{2}\right)^{\prime \prime}\right) \subset X$, then $B_{e}\left(C_{0}\left(S_{1}\right)^{\prime \prime} \times C_{0}\left(S_{2}\right)^{\prime \prime}\right) \subset Y$.

THEOREM 6.13. - Let $Y$ be a closed linear subspace of $X$ and $B: O_{0}\left(S_{1}\right) \times O_{0}\left(S_{2}\right) \rightarrow X$ a bounded bilinear operator whose range is contained in $Y$. Then the six equivalent conditions in Theorem 6.9 hold for $B$ if and only if they hold for $B$ regarded as a mapping into $Y$.

Proof. - This is an easy consequence of Theorem 6.9 and the above lemma.

\section{7. - Remarks on integration with respect to bounded bilinear operators.}

We retain the notational conventions of the previous section. Let $\beta: \mathscr{B}_{1} \times \mathscr{B}_{2} \rightarrow X$ be a separately regular vector bimeasure and $B=B_{\beta}: C_{0}\left(S_{1}\right) \times C_{0}\left(S_{2}\right) \rightarrow X$ the bounded bilinear operator corresponding to it as in Theorem 6.9. The purpose of this section is to make some comments on the possibility of replacing condition (i) in Definition 5.2 by a condition which involves the weakly compact operators $B(\cdot, k)$ and $B(h, \cdot)$ for $k \in C_{0}\left(S_{2}\right), h \in C_{0}\left(S_{1}\right)$, and the corresponding regular vector measures in place of $\beta(\cdot, F)$ and $\beta(E, \cdot)$ for $E \in \mathscr{B}_{1}, F \in \mathcal{B}_{2}$. We shall first deal with the scalar case.

LEMMA 7.1. - Let $b: C_{0}\left(S_{1}\right) \times C_{0}\left(S_{2}\right) \rightarrow K$ be a bounded bilinear form and $\beta$ the separately regular bimeasure defined by $\beta(E, F)=b_{e}\left(\chi_{E}^{\prime}, \chi_{F}^{\prime}\right), E \in \mathfrak{B}_{1}, F \in \mathfrak{B}_{2}$ (cf. Lemma 6.5). For a function $f: S_{1} \rightarrow \boldsymbol{K}$ the following three conditions are equivalent:

(i) $f$ is $\beta(\cdot, F)$-integrable for all $F \in \mathfrak{B}_{2}$;

(ii) $f$ is integrable with respect to the vector measure $\beta_{1}: \Re_{1} \rightarrow M\left(S_{2}\right)$ (cf. Theorem 4.4);

(iii) $f$ is integrable with respect to $b(\cdot, k) \in M\left(S_{1}\right)$ for all $k \in C_{0}\left(S_{2}\right)$. 
Proof. - By Lemma 5.5, (ii) follows from (i) (note that $M\left(S_{2}\right)=\operatorname{rca}\left(\mathscr{S}_{2}, \mathscr{B}_{2}\right)$ is a closed subspace of $c a\left(S_{2}, \mathfrak{B}_{2}\right)$ ). Clearly, (ii) implies both (i) and (iii) (observe that $b(\cdot, k)$ is just $k^{\prime} \circ \beta_{1}$, because $\beta_{1}$ is regular by the discussion preceding Lemma 6.1, and $\int_{\beta_{1}} h d\left(k^{\prime} \circ \beta_{1}\right)=\left\langle\int_{\mathcal{S}_{1}} h d \beta_{1}, k\right\rangle=b(h, k)$ for $h \in C_{0}\left(S_{1}\right)$ by Lemma 6.5). Assume now (iii). We shall prove (ii). This could be done by using some considerations in [20, pp. 132-133, 144], but we give a direct argument. For $n \in N$, we define $f_{n}=f_{\chi_{A_{n}}}$ where $A_{n}=\left\{s \in S_{1}:|f(s)| \leqslant n\right\}$. Then $f_{n}$ is $b(\cdot, k)$-measurable (i.e. measurable with respect to the Lebesgue extension of $\Re_{1}$ relative to $\left.b(\cdot, k)\right)$, hence $b(\cdot, k)$-integrable $\left[6\right.$, p. 117], for all $k \in O_{0}\left(S_{2}\right)$, and so are $f_{n} h$ and $f h$ for all $h \in C_{0}\left(S_{1}\right)$. From the Lebesgue dominated convergence theorem $[6, p .151]$ it follows that

$$
\lim _{n \rightarrow \infty} \int_{S_{1}} f_{n} h d b(\cdot, k)=\int_{S_{1}} f h d b(\cdot, k) \text {. }
$$

Since $(h, k) \mapsto \int_{S_{1}} f_{n} h d b(\cdot, k)$ is for every $n \in N$ a bounded bilinear form on $C_{0}\left(\mathbb{S}_{1}\right) \times$ $\times C_{0}\left(S_{2}\right)$, the uniform boundedness principle can be used to show that the bilinear form $b_{f}: C_{0}\left(S_{1}\right) \times C_{0}\left(S_{2}\right) \rightarrow \boldsymbol{K}$ defined by $b_{f}(h, k)=\int_{S_{1}} t h d b(\cdot, k)$ is bounded. Let $T: C_{0}\left(S_{1}\right) \rightarrow$ $\rightarrow C_{0}\left(S_{2}\right)^{\prime}$ be the bounded bilinear operator defined by $\langle T h, k\rangle=b_{f}(h, k)$. As $T$ is weakly compact (see the proof of Lemma 6.3), there is by Lemma 6.1 a regular vector measure $\nu: \Re_{1} \rightarrow C_{0}\left(S_{2}\right)^{\prime}$ such that $b_{f}(h, k)=\left\langle\int_{S_{1}} h d v, k\right\rangle, h \in C_{0}\left(S_{1}\right), k \in C_{0}\left(S_{2}\right)$. Writing $\lambda_{k}(E)=\int_{E} f d b(\cdot, k)$ we have $k^{\prime} \circ \nu(E)=\lambda_{k}(E)$ for all $E \in \mathscr{B}_{1}, k \in C_{0}\left(S_{2}\right)$, because $\lambda_{k}$, being $b(\cdot, k)$-continuous $\left[6\right.$, p. 114], is a regular measure, and $\int_{S_{1}} h d\left(k^{\prime} \circ \nu\right)=$ $=b_{f}(h, k)=\int_{S_{1}} h d \lambda_{k}$ for all $h \in C_{0}\left(S_{1}\right)[6, \mathrm{p} .180]$. Since

$$
\int_{E_{n}}|f(s)| v\left(k^{\prime} \circ \beta_{1}, d s\right)=\int_{E_{n}}|f(s)| v(b(\cdot, k), d s)=v\left(k^{\prime} \circ v, E_{n}\right)
$$

by Theorem $20(a)$ in $\left[6\right.$, p. 114] (recall that $b(\cdot, k)$ is identified with $k^{\prime} \circ \beta_{1}$ ), we have

$$
\sup \left\{\int_{E_{n}}|f(s)| v\left(k^{\prime} \circ \beta_{1}, d s\right): k \in C_{0}\left(S_{2}\right),|k| \leqslant 1\right\} \leqslant\|v\|\left(E_{n}\right) \rightarrow 0
$$
as $n \rightarrow \infty$ for every decreasing sequence $\left(E_{n}\right)$ of members of $\mathfrak{B}_{1}$ with $\bigcap_{n=1}^{\infty} E_{n}=\emptyset$,
and so (ii) follows from Theorem 3.5.

The following theorem is an easy consequence of the above lemma. In preparation, observe that if e.g. $f: S_{1} \rightarrow \boldsymbol{K}$ is $b(\cdot, k)$-integrable for all $k \in C_{0}\left(S_{2}\right)$, the linear functional $b(f, \cdot)$ for which

$$
b(f, k)=\int_{S_{1}} f d b(\cdot, k), \quad k \in C_{0}\left(S_{2}\right),
$$


belongs to $M\left(S_{2}\right)$; by Lemma 7.1 and its proof we have actually

$$
b(f, k)=\left\langle\int_{S_{1}} f d \beta_{1}, k\right\rangle, \quad k \in C_{0}\left(S_{2}\right) .
$$

We define $b(\cdot, g)$ analogously.

THEOREM 7.2. - Let b: $O_{0}\left(S_{1}\right) \times{O_{0}}_{0}\left(\mathscr{S}_{2}\right) \rightarrow \boldsymbol{K}$ and $\beta: \mathfrak{B}_{1} \times \mathfrak{B}_{2} \rightarrow \boldsymbol{K}$ be as in Lemma 7.1. The pair $(f, g)$ of functions $f: S_{1} \rightarrow \boldsymbol{K}, g: S_{2} \rightarrow \boldsymbol{K}$ is $\beta$-integrable if and only if the following three conditions hold:

(i) $f$ is $b(\cdot, k)$-integrable for all $k \in O_{0}\left(S_{2}\right)$, and $g$ is $b(h, \cdot)$-integrable for all $h \in C_{0}\left(S_{1}\right)$;

(ii) $f$ is $b(\cdot, g)$-integrable and $g$ is $b(f, \cdot)$ integrable;

(iii) $\int_{S_{1}} f d b(\cdot, g)=\int_{S_{\mathrm{s}}} g d b(f, \cdot)$.

If this is the case, both sides in (iii) are equal to $\int(f, g) d \beta$.

REMaRK 7.3. - Lemma 7.1 becomes false, if in (iii) $C_{0}\left(S_{2}\right)$ is replaced by $\pi\left(S_{2}\right)$, the set of continuous functions with compact support. For example, let $S_{1}=S_{2}$ be the half-open interval ]0,1], and define $b: C_{0}\left(S_{1}\right) \times C_{0}\left(S_{2}\right) \rightarrow \boldsymbol{R}$ by $b(h, k)=\int_{0}^{1} h(x)$. $\cdot k(x) d x$. The function $f: S_{1} \rightarrow \boldsymbol{R}, f(x)=x^{-2}$, is $b(\cdot, k)$-integrable for all $k \in \mathcal{K}\left(S_{2}\right)$, but not $b(\cdot, k)$-integrable, if $k(x)=x, x \in] 0,1]$. Moreover, if $g(x)=x^{2}$, the pair $(f, g)$ is $b$-integrable in the sense of $[15, \mathrm{p} .482]$ and $[20, \mathrm{p} .145]$, but not $\beta$-integrable in the sense of our definition (in this case $\beta(E, F)=m(E \cap F)$, where $m$ is the Lebesgue measure on $] 0,1])$. In $[17$, p. 23], a pair $(f, g)$ of functions satisfying the conditions (i), (ii) and (iii) of Theorem 7.2 is said to be "strongly" integrable with respect to $b$.

We now turn to the question, to what extent analogues of Lemma 7.1 and Theorem 7.2 are true in the vector case. Part of Lemma 7.1 can be easily generalized without any restriction on the Banach space $X$. In the next lemma we assume that $\beta \in \operatorname{src} a\left(\mathbb{S}_{1}, \mathscr{B}_{1} ; S_{2}, \mathscr{B}_{2} ; X\right)$ and let $B: O_{0}\left(S_{1}\right) \times O_{0}\left(S_{2}\right) \rightarrow X$ be the bounded bilinear operator defined by $B(h, k)=\int(h, k) d \beta$. By Theorem 6.9 and Remark 6.10 the operators $B(\cdot, k): C_{0}\left(S_{1}\right) \rightarrow X$ and $B(h, \cdot): C_{0}\left(S_{2}\right) \rightarrow X$ are weakly compact, $k \in O_{0}\left(S_{2}\right), h \in O_{0}\left(S_{1}\right)$.

LEMMA 7.4. - If the function $f: S_{1} \rightarrow \boldsymbol{K}$ is $\beta(\cdot, F)$-integrable for all $F \in \mathcal{B}_{2}$, then for every $k \in C_{0}\left(S_{2}\right)+$ is integrable with respect to the regular vector measure on $\Re_{1}$ corresponding to $B(\cdot, k)$ by Lemma 6.1 .

Proof. - By Theorem 5.6, $f$ is integrable with respect to the vector measure $E \mapsto \int_{s_{2}} k d \beta(E, \cdot), E \in \mathfrak{B}_{1}$. But this is just the regular vector measure corresponding to $B(\cdot, k)$, because the value of the latter for $E \in \mathfrak{B}_{1}$ is $B(\cdot, k)^{n}\left(\chi_{E}^{\prime}\right)$, which equals $B_{e}\left(\chi_{E}^{\prime}, k^{\prime}\right)$ by Lemma 6.7, and clearly $B_{e}\left(\chi_{e}^{\prime}, k^{\prime}\right)=\int_{\delta_{\mathbf{a}}} k d \beta(E, \cdot)$. 
Following E. THomas $[20$, p. 135] we say that a Banach space $X$ is weakly $\Sigma$-complete, if for every sequence $\left(x_{n}\right)$ of elements of $X$ such that $\sum_{n=1}^{\infty}\left|\left\langle x_{n}, x^{\prime}\right\rangle\right|<\infty$ for all $x^{\prime} \in X^{\prime}$ there is $x \in X$ such that $\left\langle x, x^{\prime}\right\rangle=\sum_{n=1}^{\infty}\left\langle x_{n}, x^{\prime}\right\rangle$ for all $x^{\prime} \in X^{\prime}$ (or in view of the Orlicz-Pettis theorem, equivalently, $\left.x=\sum_{n=1}^{\infty} x_{n}\right)$. In the next lemma we collect some well-known characterizations of weakly $\Sigma$-complete Banach spaces.

LemMa 7.5. - For a Banach space $X$ the following four conditions are equivalent:

(i) $X$ is weakly $\Sigma$-complete;

(ii) $X$ does not contain any isomorphie copy of $c_{0}$;

(iii) for every locally compact Hausdorff space $S$ every bounded linear operator $T: C_{0}(S) \rightarrow X$ is weakly compact;

(iv) for every set $S \neq \emptyset$, every $\sigma$-algebra $\Sigma$ of subsets of $S$ and every vector measure $\mu: \Sigma \rightarrow X$ the following holds: if $f: S \rightarrow \boldsymbol{K}$ is a function which is $x^{\prime} \mu$-integrable for all $x^{\prime} \in X^{\prime}$, then $f$ is $\mu$-integrable.

Proof. - The equivalence of (i) and (ii) is due to C. BEsSAga and A. PefdzyńsK' (see [2, p. 160]). As to the equivalence of (i) and (iii), see [18, p. 219] and [20 pp. 135-136]. Assume now (i). To prove (iv), one may observe that if $f$ is $x^{\prime} \mu$-integrable for all $x^{\prime} \in X^{\prime}$, then some $\Sigma$-measurable function agrees with $f \mu$-a.e. (see Theorem 3.2 and $[19$, p. 145]); then apply Theorem 1 in $[10$, p. 31] and Corollary 3.6. Finally, assume (iv). We prove (ii). Suppose, to the contrary, that there is a linear injection $\alpha: c_{0} \rightarrow X$ which is a homeomorphism onto its range. Take $S=N$ and let $\Sigma$ be the set of all subsets of $N$. Define $f(n)=n$ and $g(n)=n^{-1}$ for $n \in N$. Clearly, the set function $\mu: \Sigma \rightarrow X$ defined by $\mu(A)=\alpha\left(\chi_{A} g\right)$ is an $X$-valued vector measure. As the dual of $c_{0}$ is $l^{1}$, it is easy to verify that $f$ is $x^{\prime} \mu$-integrable for all $x^{\prime} \in X^{\prime}$. But $f$ is not $\mu$-integrable. This contradiction proves (ii).

CoRollari 7.6. - A Banach space $X$ is weakly $\Sigma$-complete, if and only if for all locally compact Hausdorff spaces $S_{1}$ and $S_{2}$ every bounded bilinear operator $B: C_{0}\left(S_{1}\right) \times$ $\times O_{0}\left(S_{2}\right) \rightarrow X$ satisfies the condition $B_{e}\left(O_{0}\left(S_{1}\right)^{\prime \prime} \times C_{0}\left(S_{2}\right)^{\prime \prime}\right) \subset X$.

Proof. - Let $X$ be weakly $\Sigma$-complete. By Lemma 6.7 and the weak compactness of $B(\cdot, k): C_{0}\left(S_{1}\right) \rightarrow X$ (Lemma 7.5) $B_{e}\left(\varphi, k^{\prime}\right) \in X$ for all $k \in C_{0}\left(S_{2}\right), \varphi \in C_{0}\left(S_{1}\right)^{\prime \prime}$ [6, p. 482]. Fix $\varphi \in O_{0}\left(S_{1}\right)^{\prime \prime}$. Again by Lemma 6.7 and the weak compactness of the operator $k \mapsto B_{e}\left(\varphi, k^{\prime}\right)$ from $C_{0}\left(S_{2}\right)$ to $X, B_{e}(\varphi, \psi) \in X$ for all $\psi \in C_{0}\left(S_{2}\right)^{\prime \prime}$. Suppose, conversely, that $X$ is not weakly $\Sigma$-complete. Then there is a linear injection $\alpha: c_{0} \rightarrow X$ which is a homeomorphism onto its range. Let $B: c_{0} \times c_{0} \rightarrow c_{0}$ be the bilinear operator considered in Remark 6.10. Using Theorem 6.13 we see that $\alpha_{\circ} B$ does not satisfy the condition $(\alpha \circ B)_{e}\left(C_{0}\left(\mathscr{S}_{1}\right)^{\prime \prime} \times C_{0}\left(\mathcal{S}_{2}\right)^{n}\right) \subset X$. 
In the next theorem we make the assumption that $X$ is weakly $\Sigma$-complete. By identifying a bounded operator (which is weakly compact by Lemma 7.5) from $C_{0}\left(S_{j}\right)$ to $X$ with the corresponding regular vector measure (Lemma 6.1), and defining $B(\cdot, g)$ and $B(f, \cdot)$ in a natural way, we can formulate a generalization of Theorem 7.2. (Specifically, if e.g. $f: S_{1} \rightarrow \boldsymbol{K}$ is $B(\cdot, k)$-integrable for all $k \in C_{0}\left(S_{2}\right)$, the discussion preceding Theorem 7.2 shows that for all $x^{\prime} \in X^{\prime}$ the functional

$$
k \mapsto\left\langle x^{\prime}, \int_{S_{1}} f d B(\cdot, k)\right\rangle=\int_{S_{1}} f d\left(o^{\prime} \cdot B(\cdot, k)\right)
$$

on $C_{0}\left(S_{2}\right)$ is bounded, and so by the uniform boundedness principle $k \mapsto \int_{S_{1}} f d B(\cdot, k)$ is a bounded linear operator from $C_{0}\left(S_{2}\right)$ to $X$. We denote this operator by $B(f, \cdot)$ and define $B(\cdot, g)$ similarly.)

THeoRem 7.7. - Let $X$ be a weakly $\Sigma$-complete Banach space. Suppose $\beta \in$ srca. $\cdot\left(S_{1}, \Re_{1} ; S_{2}, \Re_{2} ; X\right)$ and let $B=B_{\beta}: O_{0}\left(S_{1}\right) \times C_{0}\left(S_{2}\right) \rightarrow X$ be defined as in Theorem 6.9 . The pair $(f, g)$ of functions $f: \mathbb{S}_{1} \rightarrow \boldsymbol{K}, g: S_{2} \rightarrow \boldsymbol{K}$ is $\beta$-integrable if and only if the following three conditions hold:

(i) $f$ is $B(\cdot, k)$-integrable for all $k \in C_{0}\left(S_{2}\right)$, and $g$ is $B(h, \cdot)$-integrable for all $h \in C_{0}\left(S_{1}\right)$;

(ii) $f$ is $B(\cdot, g)$-integrable and $g$ is $B(f, \cdot)$-integrable;

(iii) $\int_{S_{1}} f d B(\cdot, g)=\int_{S_{2}} g d B(f, \cdot)$.

If this is the ease, both sides of (iii) are equal to $\int(f, g) d \beta$.

Proof. - Since $x^{\prime} \circ \beta(E, F)=\left(x^{\prime} \circ B\right)_{e}\left(\chi_{E}^{\prime}, \chi_{F}^{\prime}\right)$ for all $x^{\prime} \in X^{\prime}, E \in \mathfrak{B}_{1}, F \in \mathfrak{B}_{2}$, the theorem is an easy consequence of Theorem 7.2 and Lemma 7.5.

We conclude by showing that the restriction made on $X$ in the above theorem is the right one.

THEOREM 7.8. - For a Banach space $X$ the following two conditions are equivalent:

(i) $X$ is weakly $\Sigma$-complete;

(ii) for all locally compact Hausdorff spaces $S_{1}$ and $S_{2}$, and for all bounded bilinear operators $B: C_{0}\left(S_{1}\right) \times C_{0}\left(S_{2}\right) \rightarrow X$ satisfying the six equivalent conditions in Theorem 6.8 the following holds: if for all $k \in C_{0}\left(S_{2}\right) f: S_{1} \rightarrow \boldsymbol{K}$ is integrable with respect to the regular vector measure on $\mathfrak{B}_{1}$ corresponding to the weakly compact operator $B(\cdot, k): C_{0}\left(S_{1}\right) \rightarrow X$, then $f$ is $\beta_{B}(\cdot, F)$-integrable for every $F \in \mathfrak{J}_{2}$, where $\beta_{B}$ is defined by $\beta_{B}(E, F)=B_{e}\left(\chi_{E}^{\prime}, \chi_{F}^{\prime}\right), E \in \Re_{1}, F^{\prime} \in \Re_{2}$.

Proof. - By Theorem 7.6, (i) implies (ii). Suppose now that $X$ is not weakly $\Sigma$-complete. We shall construct a bounded bilinear operator $B: c_{0} \times c_{0} \rightarrow c_{0}$ satisfying the conditions in Theorem 6.8 and such that the statement in (ii) is not true of $B$. 
Since $X$ contains an isomorphic copy of $c_{0}$ (Lemma 7.5), it will then, in view of Lemma 6.11 and Theorem 6.13, be clear that (ii) does not hold for $X$. Let $\varphi: N \rightarrow K$ be defined by $\varphi(n)=n^{-1}$. If $h, k \in c_{0}=C_{0}(N)$, we define $B(h, k) \in c_{0}$ as the pointwise product $\varphi \hbar k$. When $l^{\infty}$ is identified in the usual way with the bidual of $c_{0}$, it is seen as in Remark 6.10 that $B_{e}(f, g)=\varphi f g$ for all $f, g \in l^{\infty}$. Thus $B_{e}\left(c_{0}^{\prime \prime} \times c_{0}^{\prime \prime}\right) \subset c_{0}$. Now, if $\psi(n)=n, n \in \boldsymbol{N}$, it is easily verified that for all $k \in c_{0} \psi$ is integrable with respect to the vector measure corresponding to $B(\cdot, k)$, but $\psi$ is not integrable with respect to $E \mapsto B_{e}\left(\chi_{E}^{\prime}, \chi_{F}^{\prime}\right)$.

\section{REFERENCES}

[1] R. G. Bartle - N. Dunford - J. T. Schwartz, Weak compactness and vector measures, Canad. J. Math., 7 (1955), pp. 289-305.

[2] C. Bessaga - A. PexczyńsKa, On bases and unconditional convergenee of series in Banach spaces, Studia Math., 17 (1958), pp. 151-164.

[3] J. K. BRooks - P. W. Lewis, Linear operators and vector measures, Trans. Amer. Math. Soc., 192 (1974), pp. 139-162.

[4] N. Dinculeanu - I. Kluvánek, On vector measures, Proe. London Math. Soc., (3) 17 (1967), pp. 505-512.

[5] M. DUсно̆̌, On vector measures in Cartesian products, Mat. Casopis Sloven. Akad. Vied, 21 (1971), pp. 241-247.

[6] N. DUnford - J. T. Schwartz, Linear operators, - I: General theory, Pure and Appl. Math., Vol. 7, Interscience, New York, 1958.

[7] M. Fréchet, Sur les fonctionnelles bilinéaires, Trans. Amer. Math. Soc., 16 (1915), pp. $215-234$.

[8] A. GRothendeck, Sur les applications linéaires faiblement compactes d'espaces àu type $O(K \bar{K})$, Canad. J. Math., 5 (1953), pp. 129-173.

[9] I. KuUVÁNEK, Characterizations of Fourier-Stieltjes transforms of vector and operator valued measures, Czechoslovak Math. J., 17 (92) (1967), pp. $261-277$.

[10] I. KuUváneK - G. KNowles, Vector measures and control systems, North Holland Mathematies Studies 20, North Holland, Amsterdam-Oxford, 1975.

[11] D. R. LEwIS, Integration with respect to vector measures, Pacific J. Math., 33 (1970), pp. 157-165.

[12] M. Monse, Bimeasures and their integral extensions, Ann. Mat. Pura Appl., (4) 39 (1955), pp. 345-356.

[13] M. Morse - W. Transue, Integral representations of bilinear functionals, Proc. Nat. Acad. Sci. U.S.A., 35 (1949), pp. 136-143.

[14] M. Morse - W. Transue, O-bimeasures $A$ and their superior integrals $A^{*}$, Rend. Circ. Mat. Palermo, (2) 4. (1955), pp. 270-300 (1956).

[15] M. Monse - W. Transue, O.bimeasures $A$ and their integral extensions, Ann. of Math., (2) 64 (1956), pp. 480-504.

[16] M. MORSE - W. Transue, The representation of a bimeasure on a general rectangle, Proc. Nat. Acad. Sci. U.S.A., 42 (1956), pp. 89-95.

[17] H. NrEMr, Stochastic processes as Fourier transforms of stochastic measures, Ann. Acad. Sci. Fenn., Ser. A I, 591 (1975), pp. 1-47.

[18] A. Paxczxískr, Projections in certain Banach spaces, Studia Math., 19 (1960), pp. 209-228. 
[19] W. Rudis, Real and complex analysis, McGraw-Hill Book Co., New York-Toronto, Ont.London, 1966.

[20] E. Thomas, L'intégration par rapport à une mesure de Radon vectorielle, Ann. Inst. Fourier (Grenoble), 20:2 (1970), pp. 55-191.

[21] K. YurNEN, Fourier transforms of noncommutative analogues of vector measures and bimeasures with applications to stochastic processes, Ann. Acad. Sci. Fenn., Ser. A. I, 1 (1975), pp. 355-385. 